\title{
Sudden diffusion of turbulent mixing layers in weakly coupled plasmas under compression
}

\author{
Giovanni Viciconte \\ CEA, DAM, DIF, F-91297 Arpajon, France \\ and LMFA UMR5509_CNRS, Université de Lyon, École centrale de Lyon, Université Claude Bernard Lyon 1, INSA Lyon, Écully, France \\ Benoît-Joseph Gréa $\odot$ \\ CEA, DAM, DIF, F-91297 Arpajon, France \\ Fabien S. Godeferd \\ LMFA UMR5509-CNRS, Université de Lyon, École centrale de Lyon, Université Claude Bernard Lyon 1, INSA Lyon, Écully, France \\ Philippe Arnault and Jean Clérouin \\ CEA, DAM, DIF, F-91297 Arpajon, France
}

(Received 18 July 2019; revised manuscript received 31 October 2019; published 13 December 2019)

\begin{abstract}
The rapid growth of viscosity driven by temperature increase in turbulent plasmas under compression induces a sudden dissipation of kinetic energy, eventually leading to the relaminarization of the flow [Davidovits and Fisch, Phys. Rev. Lett. 116, 105004 (2016)]. The interdiffusion between species is also greatly enhanced, so that mixing layers appearing at interfaces between different materials are subjected to strong dynamical modifications. The result is a competition between the vanishing turbulent diffusion and the expanding plasma microscopic diffusion. In direct numerical simulations with conditions relevant to inertial confinement fusion, we evidence regimes where compressed spherical mixing layers are quickly diffused during the relaminarization process. Using one and two-point turbulent statistics, we also detail how mixing heterogeneities are smoothed out.
\end{abstract}

DOI: 10.1103/PhysRevE.100.063205

\section{INTRODUCTION}

In inertial confinement fusion (ICF) capsules, weakly coupled plasmas produced by compressions experience a tremendous growth of the dynamic viscosity $\eta$, principally induced by a larger mean free path of charged particles as the temperature $T$ increases. In pure deuterium-tritium (DT) fuel for instance, this results from the typical power law, $\eta \sim T^{5 / 2}[1]$. Considering the small dimensions of hot spots in ICF devices, $10-100 \mu \mathrm{m}$, and the temperatures achieved, possibly above $10 \mathrm{keV}$, hydrodynamic instabilities can be partially damped [2] and small-scale velocity structures can be significantly dissipated by viscous effects [3]. Davidovits and Fisch [4] have proposed and studied the compression of a homogeneous isotropic turbulent plasma as a paradigm for sudden viscous dissipation. By rapidly converting the kinetic energy of turbulent motions into internal energy, the hot-spot temperature can be enhanced, increasing the fusion reactions rates [4]. Campos and Morgan [5] have performed fully compressible simulations showing that this mechanism depends strongly on the initial fluctuating Mach number and may not be so effective in increasing the temperature for existing ICF configurations. The concept proposed by Davidovits and Fisch [4] would indeed require supersonic turbulence. The different regimes and scaling laws characteristic of compressed turbulent plasmas have been explored theoretically and using a spectral Eddy Damped Quasi-normal Markovian mode (EDQNM) model in Viciconte et al. [6]. This unveils an important sensitivity to initial conditions related to the distribution of energy fluctuations between length scales during the sudden viscous dissipation phase. It is also found in Davidovits and Fisch [7] that the sudden dissipation mechanism depends on the ionization state of the compressed gas. Campos and Morgan [8] have further observed sudden dissipation of mass fraction variances in multicomponent plasma.

In the simulations of inhomogeneous turbulent kinetic energy layers of ICF capsule size under compression, an increased transport of turbulence toward the center of the capsule have also been measured during the implosion [6]. These observations, although not accounting for plasma molecular diffusion, suggest that this mechanism, producing mixing of the heavy elements from the ablator into the DT fuel, may occur in ICF. Can sudden viscous dissipation come along with sudden diffusion? Mixing of DT with high atomic number elements, is indeed an important issue in ICF having deleterious cooling effects on the hot spot $[9,10]$. The asymmetry of the implosion [11], the presence of defects on the capsule, like fill tube [12,13] or support tent [14] are large-scale mechanisms known as principally responsible for mixing in ICF targets. Other contributions may come from perturbations at smaller scales amplified by hydrodynamic instabilities [12]. Moreover, plasma transport coefficients have been shown to play a significant role in the mixing zone dynamics (see Vold et al. [15]). Situations where mixing is only produced by molecular diffusive processes may also occur [16,17], although in Omega capsules not designed for ignition. Actually, the question of how the mixing zone dynamics evolve with nonuniformities and strong temporal variations of plasma 
transport coefficients [18], due to temperature and mass fraction variations, is still pending.

In this work, we simulate an idealized case of the DT$\mathrm{CH}$ mixing layer in spherical compressions using conditions representative of ICF. Here the plasma transport coefficients, viscosity and diffusion, are taken into account using the pseudo-ion in Jellium (PIJ) model, proposed by Arnault [19] and validated in Ticknor et al. [18]. These simulations will be compared to simulations with constant viscosity and diffusivity to identify the importance of the transport coefficients variations on the evolution of the mixing zones and how they compete with turbulent effects.

This paper is organized as follows: After presenting the basic equations and assumptions (Sec. II), we detail the configurations used for direct numerical simulations (DNS) of DT-CH mixing layer under compression (Sec. III). We then identify the different regimes corresponding to the dominance of either turbulent or plasma diffusion on the mixing with respect to the initial Reynolds number (Sec. IV).

\section{THEORETICAL FRAMEWORK}

To describe a turbulent plasma under compression, we consider a hydrodynamic formulation similar to that used in Refs. [4,6] assuming small Knudsen numbers with the mean free path of charged particles smaller than gradients length scales. (Although the fluid approach is not always valid for some ICF targets especially at the end of the compression requiring kinetic approaches). The flow is decomposed into a base component and a perturbation one. On the one hand, the base flow accounts for the radial velocity and the DT thermodynamics quantities during the adiabatic final compression of an idealized ICF target. It can be simply derived analytically from conservation laws. On the other hand, the fluctuations account for the turbulence and the fuel or ablator mixing zone and are computed with direct numerical simulations.

\section{A. Equations for DT-CH mixtures}

We describe the DT fuel- $\mathrm{CH}$ ablator mixture using the hydrodynamics equations for momentum $\rho U_{i}$ with $i=x, y, z$, density $\rho$, mass fraction $Y \in[01]$ of $\mathrm{CH}$ (therefore $1-Y$ refers to the mass fraction of DT), and single temperature $T$. In this study, radiative effects and combustion terms due to fusion reactions are not taken into account. The variables depend on time $t$ and position $\mathbf{x}$ relative to a cartesian stationary reference frame (see for instance [15]):

$$
\begin{aligned}
\partial_{t} \rho U_{i}+\partial_{j} \rho U_{i} U_{j} & =-\partial_{i} P+\partial_{j} \tau_{i j} \\
\partial_{t} \rho+\partial_{j} \rho U_{j} & =0 \\
\partial_{t} \rho Y+\partial_{j} \rho U_{j} Y & =\partial_{j} \phi_{j}, \\
\frac{n k_{b}}{(\gamma-1)}\left(\partial_{t} T+U_{j} \partial_{j} T\right) & =-P \partial_{j} U_{j}+\partial_{j}\left(\lambda \partial_{j} T\right)+\partial_{i} U_{j} \tau_{i j},
\end{aligned}
$$

where $P$ is the pressure, $n$ the number density of particles (ions and electrons), $\gamma=5 / 3$ the heat capacity ratio, $k_{b}$ the Boltzmann constant, and $\lambda$ the temperature diffusion coefficient. In the regimes considered, i.e., weakly coupled plasmas, the equation of state can be well approximated by an ideal gas law:

$$
P=n k_{b} T \text {. }
$$

Otherwise, a polytropic behavior with $\gamma \neq 5 / 3$ is still a good local approximation. The viscous stress tensor is modelled with a Newtonian constitutive law and the diffusive flux with a Fickian law:

$$
\begin{aligned}
\tau_{i j} & =\eta\left(\partial_{j} U_{i}+\partial_{i} U_{j}-\frac{2}{3} \partial_{k} U_{k} \delta_{i j}\right), \\
\phi_{j} & =\rho \mathcal{D} \partial_{j} Y .
\end{aligned}
$$

The particularity of plasma conditions is the strong dependence of the dynamic viscosity $\eta$ and the molecular interdiffusion $\mathcal{D}$ coefficients on the temperature $T$, density $\rho$, and mass fraction $Y$ of $\mathrm{CH}$. The main objective of this work is precisely to investigate how the brutal variations of transport coefficients can influence the dynamics of turbulent mixing during the compression. Here we use the PIJ model [18-20] to predict viscosity $\eta$ and diffusion $\mathcal{D}$ across Coulomb coupling regimes from low temperatures and/or high densities to high temperatures and/or low densities. This requires us to connect results from the kinetic theory valid in the weakly coupled regime to the modeling of the strongly coupled plasmas. It extends in that respect the theory limited to kinetic regime of Refs. [21,22]. In both regimes, different trade-offs with accuracy were accepted. In the weakly coupled regime, the modeling is based on a relaxation time approximation to cope with the multicomponent issue. In the strongly coupled regime, it relies on the properties of the one component plasma (OCP) using mixing laws adapted to each transport coefficient. The connection between both regimes is performed first by extrapolating the kinetic formulas in the strongly coupled regime (with a threshold of the Coulomb logarithm) and, second, by adding the corrections from the OCP quantities that arise in excess of the kinetic contribution at large coupling. A more detailed description of the PIJ model is given in Appendix B.

In order to close the system Eqs. (1a)-(1d), we provide the mixture law for deuterium-tritium (DT) fuel and ablator $(\mathrm{CH})$ as:

$$
\frac{1}{\rho}=\frac{1-Y}{\rho_{\mathrm{DT}}}+\frac{Y}{\rho_{\mathrm{CH}}},
$$

where $\rho_{\mathrm{DT}}$ and $\rho_{\mathrm{CH}}$ are respectively the microscopic densities of the light and heavy materials. They are defined as

$$
\rho_{\mathrm{DT}}=n_{\mathrm{DT}} M_{\mathrm{DT}} \quad \text { and } \quad \rho_{\mathrm{CH}}=n_{\mathrm{CH}} M_{\mathrm{CH}}
$$

where $n_{\alpha}$ and $M_{\alpha}$ are the ion density number and atomic mass of material $\alpha$. In pure materials, we simply have $n_{\alpha}=n /(1+$ $Z_{\alpha}$ ), with $Z_{\alpha}$ corresponding to the ionization number.

At this stage and in order to solve the system of equations, it is convenient to decompose the flow quantities into base (noted with suffix $B$ ) and fluctuation (identified by small letters) parts. For instance the $i$ th velocity component is written as $U_{i}=U_{i}^{B}+u_{i}$.

\section{B. Base flow}

The objective pursued by selecting an arbitrary base flow is twofold. First, we wish to obtain simplified equations for the perturbations when expressed in a noninertial frame (see Ref. [23] for instance). Also, the thermodynamics conditions 


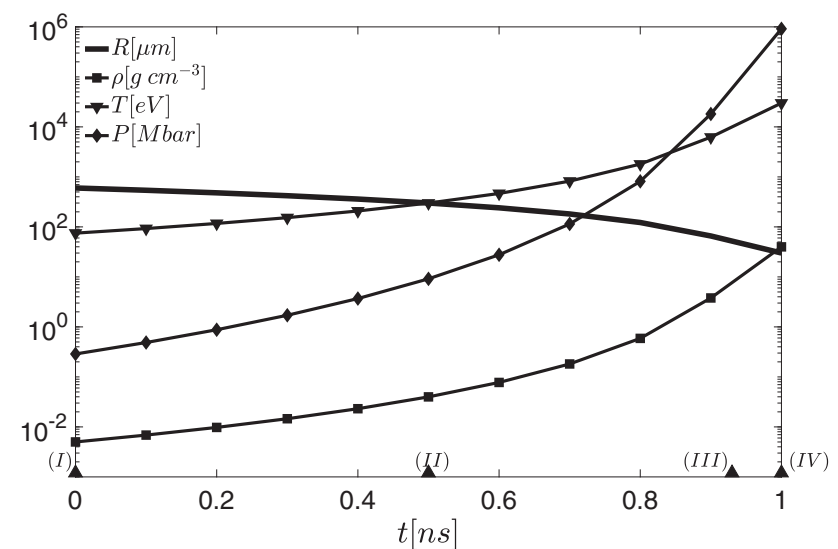

(a)

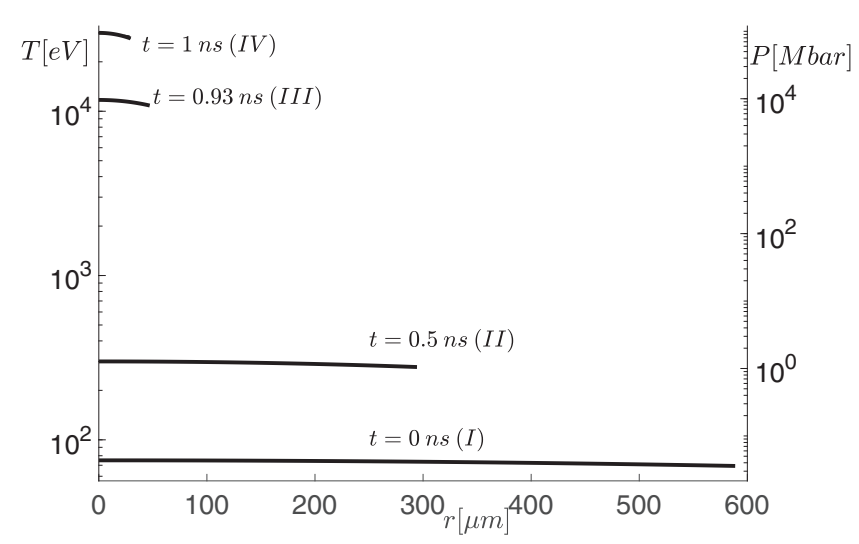

(b)

FIG. 1. Description of the implosion characteristics studied in the present work. (a) Time evolution of the temperature $T^{B}$, the pressure $P^{B}$, and density $\rho^{B}$ at the center of the domain. The thick solid line represents the radius of the capsule. (b) Radial profiles of base temperature and pressure at four instants during the compression as indicated in the left figure by the black triangles on the time axis. The instants (I), (II), (III), and (IV) are used to characterize the evolution of other quantities in the following sections.

are selected to represent the hot-spot characteristics in ICF capsules.

We start by choosing the radial base velocity field, $U_{i}^{B}$, accounting for the main compression as

$$
U_{i}^{B}(\mathbf{x}, t)=-\mathcal{S}(t) x_{i},
$$

introducing the uniform compression time rate $\mathcal{S}(t)$, assumed positive for a compression. Here the base velocity modulus grows linearly as a function of the radius $r=\|\mathbf{x}\|$ with respect to the capsule center. It is also useful to define the compression parameter, $\Lambda(t)=\exp \left(-\int_{0}^{t} \mathcal{S}(s) d s\right)$. Any nondistorted length scale evolves proportionally to $\Lambda$ during the compression. In particular, the radius $R$ corresponding to the fuel-ablator interface, if unperturbed by instabilities, would decrease as $R(t)=R_{0} \Lambda(t)$. Thereafter, initial values at $t=0$ are identified with the suffix ${ }_{0}$.

We now provide a closed analytical form for the remaining base-flow quantities. The base density $\rho^{B}$ is taken uniform and the base mass fraction is set to zero corresponding to pure DT fuel. From mass conservation these choices lead directly to:

$$
\begin{aligned}
& \rho^{B}(t)=\rho_{0} \Lambda(t)^{-3}, \\
& n^{B}(t)=n_{0} \Lambda(t)^{-3}, \\
& Y^{B}(t)=Y_{0}=0 .
\end{aligned}
$$

The base temperature $T^{B}$ can be derived from Eqs. (1d) and (2) and keeping spherical symmetry. The compressions are here assumed to be adiabatic such that all the dissipative terms coming from viscosity or thermal diffusion, but also from fluctuations, are neglected. We thus obtain:

$$
T^{B}(r, t)=T_{0} \Lambda(t)^{-3(\gamma-1)}\left(1-\frac{1}{h_{0}^{2}} \frac{r^{2}}{\Lambda(t)^{2}}\right) .
$$

Here an arbitrary quadratic profile is imposed in order to have higher temperature at the center of the domain. This form also allows to close the momentum equation as will be shown below. The length scale $h_{0}>0$ expresses the temperature gradient at the beginning of the compression. The base pressure $P^{B}$ can be simply deduced from the equation of state, leading to:

$$
P^{B}(r, t)=P_{0} \Lambda(t)^{-3 \gamma}\left(1-\frac{1}{h_{0}^{2}} \frac{r^{2}}{\Lambda(t)^{2}}\right) .
$$

An analytic expression for the compression parameter $\Lambda(t)$ can be derived from the base-flow momentum equation in this adiabatic approximation. In this calculations, it was important to have a linear radial pressure gradient to balance all the terms of the momentum equation. We thus obtain:

$$
\Lambda(t)=\sqrt{1-2 \mathcal{S}_{0} t+\left(\mathcal{S}_{0}^{2}+\Omega_{0}^{2}\right) t^{2}},
$$

with the characteristics frequencies $\mathcal{S}_{0}=\mathcal{S}(0)$, the initial compression rate, and $\Omega_{0}=\left(\frac{2 P_{0}}{\rho_{0} h_{0}^{2}}\right)^{1 / 2}$ expressing pressure gradient effects. The radial position of the fuel-ablator interface, $R(t)=R_{0} \Lambda(t)$, is principally driven by the initial impulsion $\mathcal{S}_{0}$ of the compression and then decelerated by pressure gradient effects, $\Omega_{0}$, leading to a minimum convergence radius (see Fig. 1).

The adiabatic compressions studied here differ sensibly from realistic implosions of ICF capsules which are driven by shocks. However, it is possible to choose parameters such that the duration and convergence ratio are representative of ICF implosions. In addition, we can impose at minimum convergence ratio the thermodynamics conditions relevant to hot spots in ICF capsules (see Ref. [24]). These conditions are gathered in Table I and in Fig. 1.

The initial conditions at $t=0$ correspond to a compressed and heated plasma state where DT and $\mathrm{CH}$ are already almost fully ionized with $Z_{\mathrm{DT}}=1$ and $Z_{\mathrm{CH}}=3.5$. The inner radius diameter is $R_{0}=300 \mu \mathrm{m}$, the duration of the compression $1 \mathrm{~ns}$ and a convergence ratio of 20 is achieved. The base temperature at the center of the capsule varies from $75 \mathrm{eV}$ to $30 \mathrm{keV}$, with the base pressure reaching $10^{3} \mathrm{Gbar}$ and fuel density $40 \mathrm{~g} \mathrm{~cm}^{-3}$. These values are sensitively larger than the ones encountered in realistic ICF capsules (with at most temperature $\sim 5 \mathrm{keV}$ and pressure $\sim 300 \mathrm{Mbar}$ ). In addition, 
TABLE I. Temperature $T$, pressure $P$, density $\rho$, and coupling parameter $\Gamma$ at the center of the capsule for different instants. The last column indicates the radius of the unperturbed fuel-ablator interface $R$.

\begin{tabular}{lccccc}
\hline \hline$t(\mathrm{~ns})$ & $T(\mathrm{eV})$ & $P($ Mbar $)$ & $\rho\left(\mathrm{g} \mathrm{cm}^{-3}\right)$ & $\Gamma$ & $R(\mu \mathrm{m})$ \\
\hline 0 & 75 & $2.87 \times 10^{-1}$ & $5 \times 10^{-3}$ & $3.17 \times 10^{-2}$ & 300 \\
0.5 & 300 & 9.2 & $4 \times 10^{-2}$ & $1.63 \times 10^{-2}$ & 150 \\
0.93 & $11.7 \times 10^{3}$ & $8.75 \times 10^{4}$ & 9.75 & $2.6 \times 10^{-3}$ & 24 \\
1 & $30 \times 10^{3}$ & $9.19 \times 10^{5}$ & 40 & $1.9 \times 10^{-3}$ & 15 \\
\hline \hline
\end{tabular}

we do not model the inner DT layer producing higher density at the DT-CH interface. Therefore the effects of transport coefficients are exacerbated in this configuration compared to a realistic capsule. Indeed, the objective is to investigate the phenomenology on relatively large mixing zones, in particular to extract angular spectra. But configurations closer to standard National Ignition Facility (NIF) or Omega configurations are also presented and detailed in Appendix A.

In Fig. 1, the temperature and pressure profiles at different times show the gradients responsible for the deceleration of the capsule radius. It also generates Rayleigh-Taylor instability at the fuel-ablator interface. However, due to the fact that $\Omega_{0}=50 \mu \mathrm{s}^{-1} \ll \mathcal{S}_{0}=1000 \mu \mathrm{s}^{-1}$, its effect remains weak during the implosion.

\section{Perturbation equations}

In this section, we derive the system of equations determining the dynamics of the perturbations around the base flow. Then we show how the system can be greatly simplified by working in a noninertial frame.

The perturbations equations are deduced from Eqs. (1a)(1d) after having introduced the base-flow expression presented in the previous section. We obtain:

$$
\begin{aligned}
\partial_{t} u_{i} & +u_{j} \partial_{j} u_{i}-\mathcal{S} x_{j} \partial_{j} u_{i}-\mathcal{S} u_{i} \\
= & -\partial_{i} \pi-\pi \partial_{i} \theta-\Pi^{B} \partial_{i} \theta \\
& +\partial_{j}\left[v\left(\partial_{j} u_{i}+\partial_{i} u_{j}-\frac{2}{3} \partial_{k} u_{k} \delta_{i j}\right)\right] \\
& \quad-v\left(\partial_{j} u_{i}+\partial_{i} u_{j}-\frac{2}{3} \partial_{k} u_{k} \delta_{i j} \partial_{j} \theta\right), \\
\partial_{t} \theta+ & u_{j}-\mathcal{S} x_{j} \partial_{j} \theta \\
= & \partial_{j} u_{j}, \\
\partial_{t} n & +\partial_{j}\left(n u_{j}\right)+\partial_{j}\left(n^{B} u_{j}\right)+\partial_{j}\left(n U_{j}^{B}\right) \\
= & \partial_{j}\left(\mathcal{D} \partial_{j} n\right)-\partial_{j}\left[\left(n+n^{B}\right) \mathcal{D} \partial_{j} \theta\right],
\end{aligned}
$$

where $v=\eta / \rho$ is the kinematic viscosity. Here we work with the density $\operatorname{logarithm}, \Theta=\log \left(\rho / \rho_{0}\right)$, allowing a more compact equation system within the variable density framework detailed below. This quantity is expressed by its base and fluctuating part as $\Theta=\Theta^{B}+\theta$, with $\Theta^{B}=\log \left(\rho^{B} / \rho_{0}\right)$. This shows that $\theta$ is the renormalized density logarithm, $\theta=$ $\log \left(\rho / \rho_{B}\right)$. In addition, we use the reduced pressure defined as $\Pi=\frac{P}{\rho}$ and similarly decomposed into $\Pi=\Pi^{B}+\pi$ with $\Pi^{B}=P^{B} / \rho^{B}$.

At this stage, we assume that particle density fluctuations are small, $\frac{n}{n_{B}} \ll 1$. From this assumption and Eq. (11c), it follows that

$$
\partial_{j} u_{j}=-\partial_{j} \mathcal{D} \partial_{j} \theta .
$$

This corresponds to the variable density approximation, discussed with more details in Refs. [25,26]. Therefore, compressibility is accounted only for the base flow, while it is neglected for the perturbations. The perturbation is thereafter fully described by the velocity $u_{i}$ and the density logarithm fluctuation $\theta$.

We now follow the procedure proposed in Ref. [23] in order to simplify Eqs. (11a) and (11b) through rescaling and reference frame change. We first introduce the new time $\tilde{t}$ and position variable $\tilde{\mathbf{x}}$ corresponding to the reference frame deforming with the base radial velocity:

$$
\tilde{t}=\int_{0}^{t} \Lambda^{-2}(s) d s, \quad \tilde{\mathbf{x}}=\frac{\mathbf{x}}{\Lambda(t)} .
$$

Moreover, we use the following rescaling in order to eliminate inhomogeneous forcing terms due to base velocity in the velocity equation as in Ref. [27],

$$
\begin{aligned}
\tilde{\mathbf{u}}(\tilde{\mathbf{x}}, \tilde{t}) & =\mathbf{u}(\mathbf{x}, t) \Lambda(t), \\
\tilde{\pi}(\tilde{\mathbf{x}}, \tilde{t}) & =\pi(\mathbf{x}, t) \Lambda^{2}(t), \\
\tilde{\theta}(\tilde{\mathbf{x}}, \tilde{t}) & =\theta(\mathbf{x}, t) .
\end{aligned}
$$

Finally, we obtain the following equations for the perturbations in the noninertial frame:

$$
\begin{aligned}
\partial_{t} \tilde{u}_{i}+\tilde{u}_{j} \partial_{j} \tilde{u}_{i}= & -\partial_{i} \tilde{\pi}-\tilde{\pi} \partial_{i} \tilde{\theta}-\Lambda^{2} \Pi^{B} \partial_{i} \tilde{\theta}+\partial_{j}\left[v\left(\partial_{j} \tilde{u}_{i}+\partial_{i} \tilde{u}_{j}\right)\right] \\
& -v\left(\partial_{j} \tilde{u}_{i}+\partial_{i} \tilde{u}_{j}\right) \partial_{j} \tilde{\theta}, \\
\partial_{t} \tilde{\theta}+\tilde{u}_{j} \partial_{j} \tilde{\theta}= & \partial_{j}\left(\mathcal{D} \partial_{j} \tilde{\theta}\right), \\
\partial_{j} \tilde{u}_{j}= & -\partial_{j}\left(\mathcal{D} \partial_{j} \tilde{\theta}\right) .
\end{aligned}
$$

In Eq. (15a) for the velocity perturbation, one inhomogeneous term remains which is proportional to the base reduced pressure, $\Pi^{B}$, and accounts for the buoyancy production. The quantity, $\tilde{\pi}$, expressed in the new coordinate system accounts not only for the reduced pressure fluctuations but also for the diagonal components of the viscous stress tensor. The system of Eqs. (15a)-(15c) is solved numerically by spectral methods.

\section{SIMULATION SET UP}

In this section, we first explain the numerical procedures used to solve the system for the perturbations equations. Then we detail how the initial conditions are parameterized and present the various cases investigated in this study.

\section{A. Numerical methods}

We solve the system Eqs. (15a)-(15c) in Fourier space following classical pseudospectral methods proposed in Ref. [28] and using the code developed in Ref. [6]. The cubic computational domain of $2 \pi$ size is periodic. Because of this periodicity, it is important to ensure that the perturbations corresponding to the mixing at fuel-ablator interface do not reach the boundaries. This would otherwise artificially bias the results as if mixing zones from different imploding capsules influenced each others. The simulations are advanced in time 
using a third-order Runge-Kutta total variation diminishing (TVD) scheme [29].

Contrary to classical incompressible simulations where the Poisson equation for the pressure can be solved directly, in the variable density approximation the nonlocality in spectral space requires an iterative method to invert the linear system $[26,30,31]$. We use in our code a general minimal residual (GMRES) algorithm [32], which ensures convergence even if density fluctuations are large, contrary to fixed point methods.

As stated before, the plasma transport coefficients are computed using Arnault's PIJ model [19]. Viscous and diffusive contributions are taken into account implicitly, also using a GMRES algorithm.

The code is fully parallelized with two-dimensional (2D) domain decomposition. The typical cost of a simulation is roughly $200000 \mathrm{CPU}$ h dispatched over 2048 cores.

\section{B. Initial conditions}

In order to define more precisely the initial conditions in the simulations, we introduce the volume average of a quantity $*$ as $\langle *\rangle$. It is also convenient to use $\bar{*}$ as the average of the same quantity over fluctuations at fixed radial position. The fluctuations around these radial averaged profiles are noted as $*^{\prime}$. At this stage quantities are expressed by a triple decomposition. For instance, the full velocity is given by $U_{i}=U_{i}^{B}+u_{i}=U_{i}^{B}+\overline{u_{i}}+u_{i}^{\prime}$.

In the configurations studied, the initial density profile is radial without fluctuations, such that $\theta=\bar{\theta}$, forming diffuse spherical interface between fuel and ablator of width $L_{0}=$ $5 \mu \mathrm{m}$, centered on radius $R_{0}=300 \mu \mathrm{m}$. $\theta$ is zero at the center of the capsule, while in the ablator region of pure $\mathrm{CH}$, $\theta=0.15$. This latter value is obtained from Eq. (5) as the density number of particles is constant, $n=n_{0}$, to ensure the variable density approximation. The Atwood number between DT and $\mathrm{CH}$ is then given by $\mathrm{At}=\frac{\rho_{\mathrm{CH}}-\rho_{\mathrm{DT}}}{\rho_{\mathrm{CH}}+\rho_{\mathrm{DT}}}=0.08$. Along with the small value of the mean base pressure gradient, this small Atwood number value explains why Rayleigh-Taylor instability remains limited.

Random velocity perturbations with zero mean $\left(\left\langle u_{i}\right\rangle=0\right)$ are added around the interface. To do so, we first generate an incompressible velocity field in the full domain, which is determined by a classical von Karman spectra with integral scale $\ell_{0}$ and rms fluctuations $u_{0}$ as in Ref. [6]. The spectrum is of Batchelor type, i.e., the distribution of energy at small wave number $k$ scales $\sim k^{4}$. This velocity is then filtered using a spherical shell mask in physical space localized at the diffuse interface. In order to ensure the variable density condition, Eq. (15c), we add the (small) radial compressible part provided by $\theta$.

Along with the initial size of the mixing layer $L_{0}$, the simulations are characterized by an initial Reynolds number, $\operatorname{Re}_{0}=\frac{u_{0} \ell_{0}}{v}$, here defined with the DT viscosity at the center of the capsule (see Table II). The values range from $\operatorname{Re}_{0}=0$, for simulations without turbulence, to $\mathrm{Re}_{0}=217$, reaching the limits of the available computational resources. In addition, the compression parameter $\mathrm{Cp}_{0}=\frac{u_{0}}{\ell_{0} \mathcal{S}_{0}}$ shows that the compression is relatively rapid compared to turbulence in the simulations. This choice permits us to avoid the contamination of the whole domain by mixing before the bang time. We
TABLE II. Simulation characteristics in terms of initial Reynolds number, compression number, integral length scale, and mixing layer size. Type $a$ corresponds to simulations with varying plasma transport coefficients while for type $b$ viscosity and molecular diffusion are kept constant during the computation.

\begin{tabular}{lccccccc}
\hline \hline Name & & Type & Mesh size & $\mathrm{Re}_{0}$ & $\mathrm{Cp}_{0}$ & $\ell_{0} / R_{0}$ & $L_{0} / R_{0}$ \\
\hline$S 1$ & $a$ & DNS & $512^{3}$ & 217 & 0.27 & 0.07 & 0.016 \\
& $b$ & ILES & $1024^{3}$ & 217 & 0.27 & 0.07 & 0.016 \\
$S 2$ & $a$ & DNS & $512^{3}$ & 153 & 0.2 & 0.07 & 0.016 \\
& $b$ & ILES & $512^{3}$ & 153 & 0.2 & 0.07 & 0.016 \\
$S 3$ & $a$ & DNS & $512^{3}$ & 108 & 0.13 & 0.07 & 0.016 \\
& $b$ & DNS & $512^{3}$ & 108 & 0.13 & 0.07 & 0.016 \\
$S 4$ & $a$ & DNS & $256^{3}$ & 32 & 0.1 & 0.05 & 0.016 \\
& $b$ & DNS & $256^{3}$ & 32 & 0.1 & 0.05 & 0.016 \\
$S 5$ & $a$ & DNS & $256^{3}$ & 0 & 0 & 0 & 0.016 \\
\hline \hline
\end{tabular}

note also that these parameters are not far from those found in Ref. [3] for NIF simulations where the integral scale is $\ell_{0}=100 \mu \mathrm{m}$ (we have $\ell_{0}=23 \mu \mathrm{m}$ ) and $\operatorname{Re}_{0}=10$.

At this stage, it is important to assess the validity of the variable density approximation from the initial conditions. The $S 1$ set of simulations, corresponds to the largest rms velocity $u_{0}=1.73 \mathrm{~km} \mathrm{~s}^{-1}$, and to a perturbation Mach number value of $M^{\prime}=u_{0} / c=0.02$, using the sound speed $c=$ $\left(\gamma P_{0} / \rho_{0}\right)^{1 / 2}=79 \mathrm{~km} \mathrm{~s}^{-1}$. This small perturbation Mach number ensures the validity of the variable density approximation during the whole simulation. The benefit of using the variable density approximation in our code has to be stressed. Indeed, a fully compressible code is very demanding in numerical resources and may have failed to capture the small scales of turbulence due to the shock capturing schemes [33]. Actually, the initial base velocity expressing the compression at interface radius $R_{0}$ is $300 \mathrm{~km} \mathrm{~s}^{-1}$ giving a base Mach number of $M^{B}=3.8$. This shows that energy contained in the perturbation is small compared to the base component, justifying to neglect the feedback of the perturbations on the base flow.

\section{Resolution}

A total of nine simulations are presented in this study, varying the initial Reynolds numbers and using either varying transport coefficients (type $a$ ) or constant ones (type $b$ ) in order to assess their importance as the turbulence increases (Table II).

The simulations start from an initial cubic domain of $1200 \mu \mathrm{m}$ size. This choice results from a trade-off between a large volume encompassing the fuel-ablator interface and avoiding confinement effects, and a small volume where the finest turbulent structures are correctly resolved.

Therefore, the mesh size depends on the initial Reynolds number and the simulation type $a$ or $b$. The most demanding simulations, $S 1_{b}$ and $S 2_{b}$, use $1024^{3}$ and $512^{3}$ mesh sizes, respectively. In these two simulations with constant viscosity and molecular diffusion, the small scales of turbulence are not fully resolved, and hence they enter the category of implicit large eddy simulations (ILES) [34]. All other simulations can be considered as DNS. In particular, simulations with plasma coefficients (type $a$ ) are less demanding as the diffusion and viscosity growths rapidly smooth the small scales of turbulence. 


$$
\mathrm{t}=0 \mathrm{~ns}(\mathrm{I})
$$

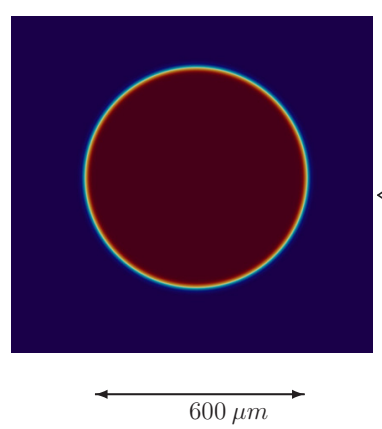

$\mathrm{t}=0.5 \mathrm{~ns}(\mathrm{II})$
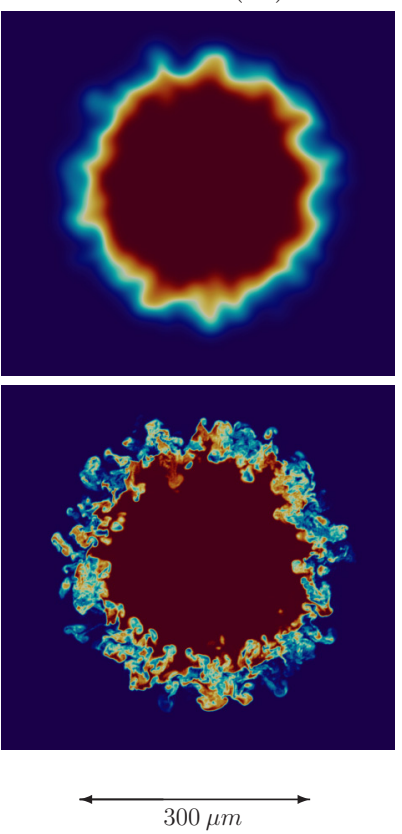

$\mathrm{t}=0.93 \mathrm{~ns}(\mathrm{III})$
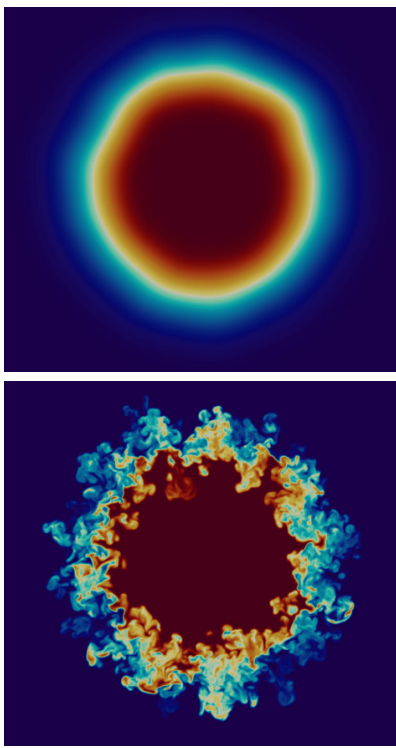

$\mathrm{Y}_{\mathrm{CH}}$ 0.000
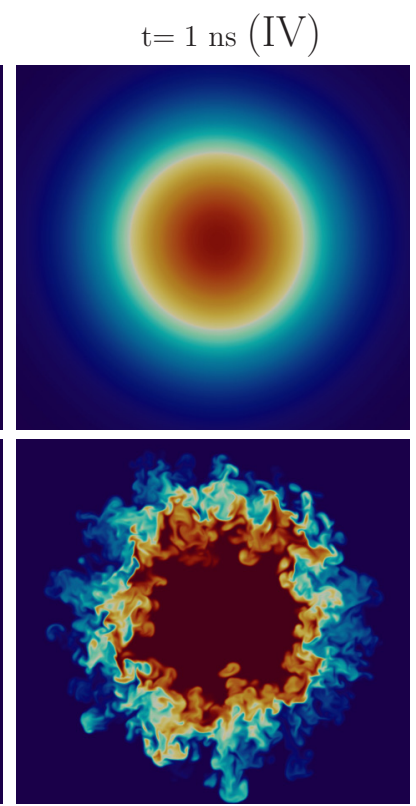

$30 \mu m$

FIG. 2. Two-dimensional contours of mass fraction $Y$ of $\mathrm{CH}$ at different instants and for simulations with varying, $S 1_{a}$, and constant, $S 1_{b}$, viscosity and diffusion coefficients. The different times correspond to those of Fig. 1.

The choice of using an initial diffuse interface of size $L_{0}=5 \mu \mathrm{m}$ is also dictated to be well resolved even for $256^{3}$ mesh sizes. We have tested different choices without deeply modifying the results presented.

Figure 2 illustrates the 3D distribution the mass fraction $Y$ of $\mathrm{CH}$ at different instants for $S 1_{a, b}$ simulations. This evidences the strong imprint of transport coefficients which dissipates turbulence during the implosion $\left(S 1_{a}\right)$. We quantify this phenomenon in the following section.

For the sake of completeness with respect to ICF conditions, we add in Appendix A results showing the mixing zone sizes for different compression rate and thermodynamics conditions more relevant to NIF or OMEGA implosions.

\section{RESULTS}

In this section, we extract the different turbulent quantities from simulations in order to shed light on the effects of plasma transport coefficients on the dynamics of turbulent layers.

\section{A. Global statistics}

Figure 3 shows the variations of the turbulent kinetic energy, $\langle\mathcal{K}\rangle=\left\langle u_{i}^{\prime} u_{i}^{\prime}\right\rangle / 2$, and of the variance $\left\langle\theta^{\prime} \theta^{\prime}\right\rangle$ for

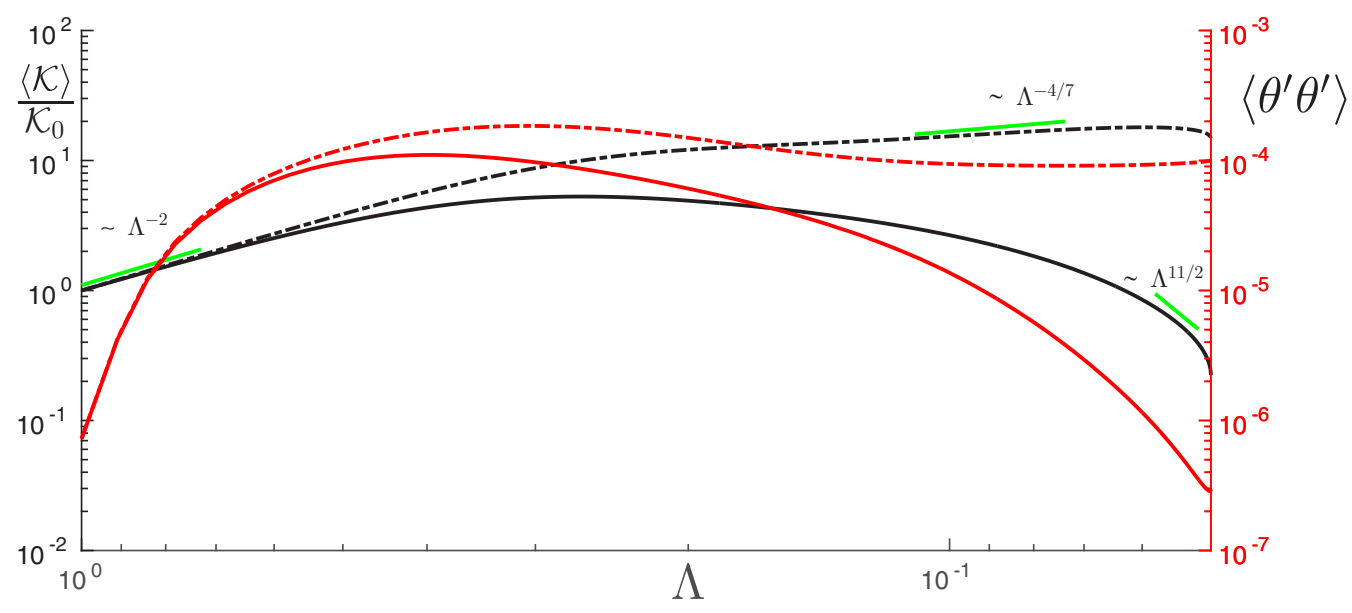

FIG. 3. Evolution of the turbulent kinetic energy normalized by its initial value (black) and the scalar variance $\left\langle\theta^{\prime} \theta^{\prime}\right\rangle$ (red) as functions of the compression parameter $\Lambda$. The solid lines represent the results of plasma transport coefficients simulation $S 1_{a}$ and the dash-dotted lines are for $S 1_{b}$ results. Scaling laws corresponding to the self-similar regimes identified in Ref. [6] are also plotted. 


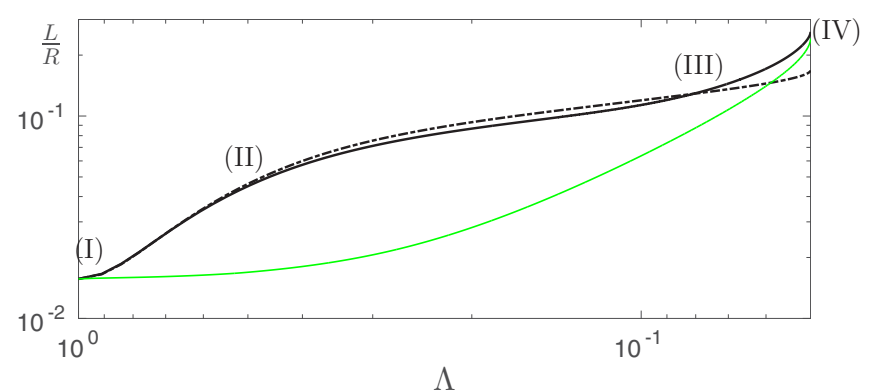

FIG. 4. Evolution of the renormalized mixing layer width as function of the compression parameter $\Lambda$. The solid black line for the case of plasma transport coefficients $\left(S 1_{a}\right)$. The black dashed line is for the constant case $\left(S 1_{b}\right)$. The solid green line is the reference case with $\operatorname{Re}=0\left(S 5_{a}\right)$, where the mixing layer is only enlarged by molecular diffusion.

high-Reynolds-number simulations $S 1_{a, b}$. This comparison shows the role of plasma transport coefficients in the mixing evolution.

For both simulations, the dynamics of the flow is first driven by compression effects, leading to an increase of kinetic energy. This phase, identified as the rapid compression in Ref. [6], leads to a $\Lambda^{-2}$ growth of $\langle\mathcal{K}\rangle$. Simultaneously, the density fluctuations initially at rest and expressed by $\left\langle\theta^{\prime} \theta^{\prime}\right\rangle$ rise rapidly due to the advection term. After this initial phase, simulations $S 1_{a}$ and $S 1_{b}$ start to differ. On the one hand, for the $S 1_{a}$ simulation, the turbulent kinetic energy growth is progressively slowed down due to the viscosity increase. Then the sudden viscous dissipation effect occurs [4], since the turbulent production mainly due to compression cannot balance viscous terms. Note that a scaling $\langle\mathcal{K}\rangle \sim \Lambda^{11 / 2}$ is expected for the self-similar decay of a Batchelor spectra with constant implosion rate [6].

On the other hand, the $S 1_{b}$ simulation goes from rapid compression to a nearly cascade regime dominated by turbulent nonlinear transfers. This phase lasts until the end of the compression with scaling close to $\Lambda^{-4 / 7}$ as expected for a Batchelor spectrum [6]. A sharp decrease is observed at the end of all the simulations, which can be attributed to the deceleration of the compressed matter.

The smaller values of $\left\langle\theta^{\prime} \theta^{\prime}\right\rangle$ in $S 1_{a}$ simulations compared to $S 1_{b}$ are the first indication of enhanced mixing due to plasma transport coefficients. The dissipation process ob- served on the scalar variance seems to confirm the observation in Ref. [8].

\section{B. Mixing layer width}

We now compare the time evolutions of the size of the mixing layers in Fig. 4 for high Reynolds simulations $S 1_{a, b}$, with or without plasma effects, and also for $S 5$ to stress the role of plasma transport coefficients when turbulence is absent.

The mixing layer sizes $L$ can be evaluated in simulations using the following integral function of the radial averaged mass fraction $\bar{Y}(r, t)[35,36]$ :

$$
L(t)=6 \int_{0}^{+\infty} \bar{Y}(t, r)[1-\bar{Y}(t, r)] d r .
$$

During the implosion, the mixing layers in $S 1_{a, b}$ simulations first experience a growth due to the rapid compression regime identified in Sec. IV A. This growth is slowed due to the dissipation of turbulent energy, either by the cascade process in $S 1_{b}$ or directly by transport coefficient in $S 1_{a}$. This process is very similar to the growth of a mixing layer in plane Richtmyer-Meschkov instability as the scales of turbulence are small compared to the radius of the interface.

Despite the active role of plasma viscosity in destroying turbulence and relaminarizing the flow, $S 1_{a}$ simulation has only a slightly lower growth rate than $S 1_{b}$ simulation until time (III) $t=0.93 \mathrm{~ns}$. This can be explained as the dynamics of a layer is principally driven by the large energetic scales, which are not dissipated by viscous effects acting at smaller scales. The molecular diffusion increase can also compensate the loss of turbulent diffusion in $S 1_{a}$ simulation. Eventually, this process completely dominates the turbulence at the end of the simulation leading to a sudden diffusion of the layer also present in $S 5$ simulation at $\operatorname{Re}=0$.

It is during this last sudden diffusion phase where $S 1_{a, b}$ simulations become significantly different, with the mixing layer width in $S 1_{a}$ increasing rapidly much above the values obtained in $S 1_{b}$ simulation.

The effect of initial conditions, mainly with varied Reynolds number detailed in Table II, is now investigated in Fig. 5. For the range of $\mathrm{Re}$ investigated, the final sizes of mixing layers is weakly sensitive to the initial level of turbulence in simulations accounting for plasma transport coefficients [Fig. 5(a)]. The difference between simulations with or without varying plasma transport coefficients mainly occurs during the sudden diffusion phase, appearing earlier in lowReynolds-number simulations. Besides, the relaminarization
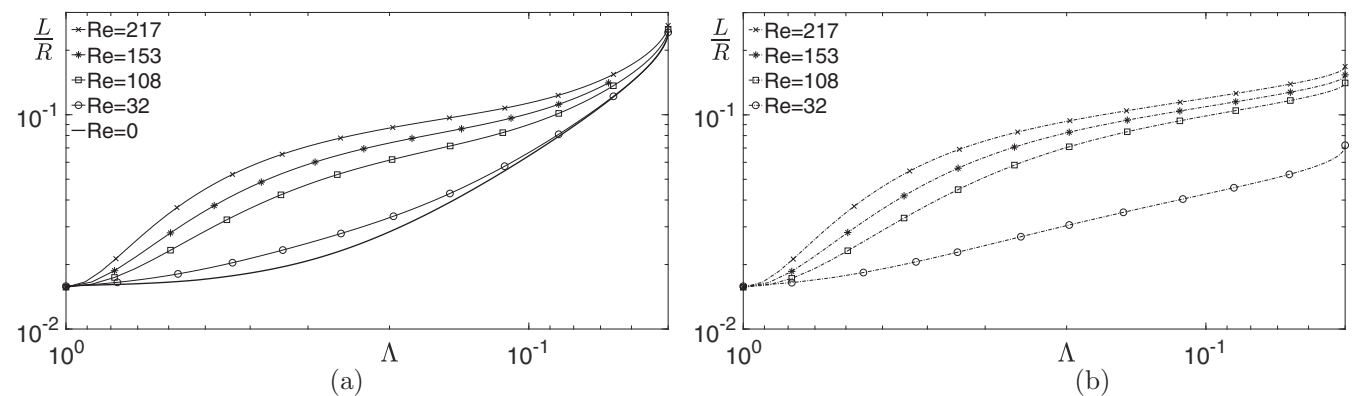

FIG. 5. Evolution of the mixing layer width as a function of the compression parameter $\Lambda$ for the simulations of Table II. (a) Simulation with plasma transport coefficients $S 1_{a}-S 5_{a}$. (b) Simulations with constant transport coefficients $S 1_{b}-S 4_{b}$. 
process due to viscosity increase has a low impact on the mixing layer dynamics. We also observe that the mixing layer widths in simulations $S 1_{b}-S 4_{b}$, shown in Fig. 5(b), during the final phase of the compression have a convex curvature implying a widening of the mixing zone not caused by transport coefficients effects. We remark that this phenomenon is taking place during the deceleration phase of the compression, suggesting that this is caused by the onset of Rayleigh-Taylor instabilities. This has been verified confronting these results with simulations where the compression parameter $\Lambda$ has a linear dependence with time and does not experience a deceleration phase (not shown).

The simulations at higher Reynolds number would allow us to determine a critical Re where the sudden diffusion no longer occurs, but these simulations are presently out of reach, as they are too computationally demanding. Moreover, the value of the critical Re should depend on the compression characteristics together with the initial value of the compression number $\mathrm{Cp}$ as expected from the study in Ref. [6] based on EDQNM simulations.

The present results focus on the impact of the initial conditions of turbulence on the diffusion process of the DT-CH interface using a hot-spot temperature of $30 \mathrm{keV}$ enhancing the conditions commonly encountered in actual ICF device. However, one can ask whether the same physics still occur with different implosion characteristics such as decreasing the hot spot's temperature or density or the compression time rate $\mathcal{S}_{0}$. The additional simulations presented in Appendix A

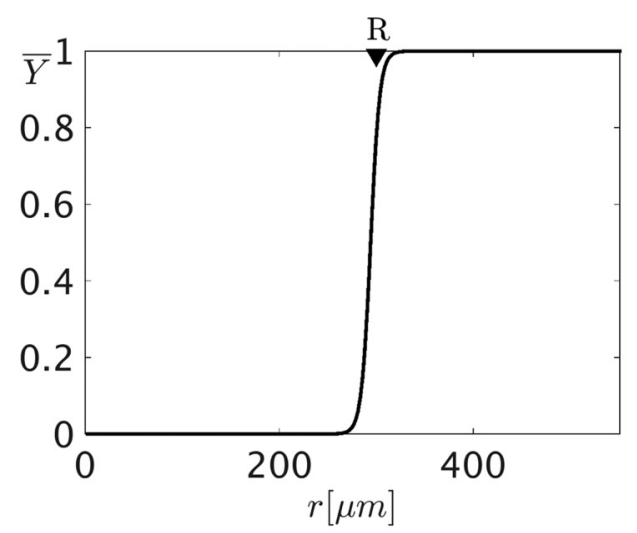

(a)

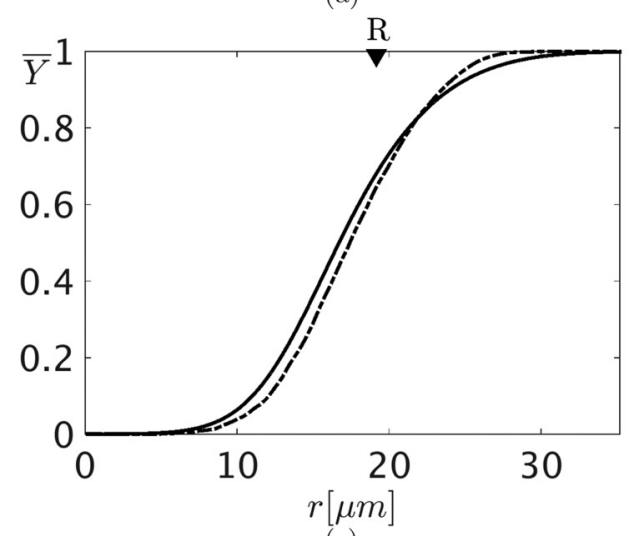

(c) give an insight of these effects. Reducing temperature leads to smaller mixing zone sizes due to much smaller values of transport coefficients (see Figs. 16 and 17). The effects of diffusion and the same phenomenology already discussed is still visible in these simulations. More precisely, we can compare directly $S 1_{a}$ and $N S 1_{a}$ simulations having same implosion characteristics except for the hot-spot temperature varying from 30 to $5 \mathrm{keV}$. This leads to renormalized mixing zones at the end of the compression varying from $L_{S 1_{a}}=$ 0.24 to $L_{N S 1_{a}}=0.07$, respectively, which can be explained as follows. Indeed, for plasma in the kinetic regime, the temperature difference implies $\left(\mathcal{D}_{S 1_{a}} / \mathcal{D}_{N S 1_{a}}\right)=(30 / 5)^{5 / 2}$. Now, using basic dimensional analysis, this gives $L_{S 1_{a}} / L_{N S 1_{a}} \sim$ $\left(\mathcal{D}_{S 1_{a}} / \mathcal{D}_{N S 1_{a}}\right)^{1 / 2} \sim 7$. The fact that $L_{N S 1_{a}}=0.07$ is slightly larger than the expected value (0.04) is due to turbulent diffusion which is not negligible as the end of NS1 simulation.

On the contrary, decreasing the compression time rate $\mathcal{S}_{0}$ (see Fig. 16) or the density, as encountered in Omega experiments (see Fig. 19), significantly enhance the mixing zone sizes.

\section{Radial profiles}

In this section, we explore the mean radial structures of mixing zones and compare their evolution when relaminarization process occurs due to plasma transport coefficients.

We show in Fig. 6 the mean mass fraction of ablator, $\bar{Y}$, extracted from $S 1_{a, b}$. The selected times correspond to the

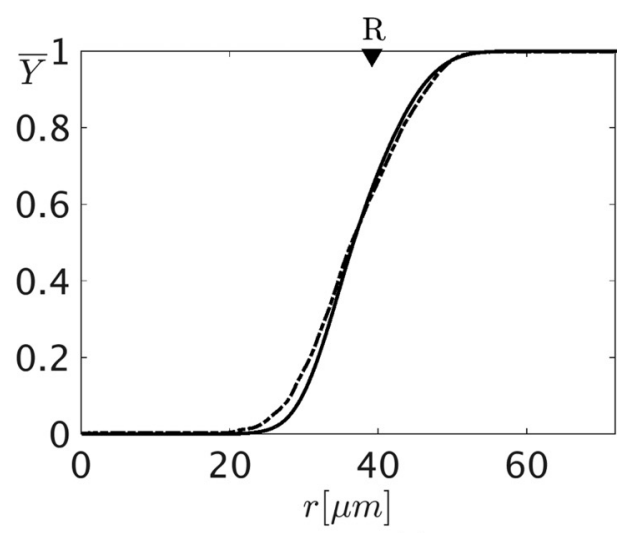

(b)

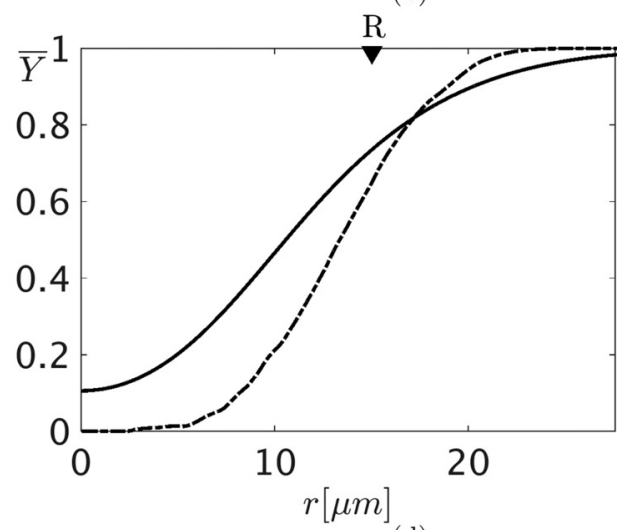

(d)

FIG. 6. Ablator (CH) tangential averaged mass fraction $Y$ at four times during compression. (a) Initial condition at $t=0 \mathrm{~ns}$ and $\Lambda=1$, (b) (II) $t=0.5 \mathrm{~ns}$ and $\Lambda=0.5$, (c) (III) $t=0.93 \mathrm{~ns}$ and $\Lambda=0.08$, and (d) (IV) $t=1 \mathrm{~ns}$ and $\Lambda=0.05$. Solid line for the $S 1_{a}$ simulation and dash-dotted line for the $S 1_{b}$ simulation. The Lagrangian position of the unperturbed fuel-ablator interface is also indicated in the figure. 


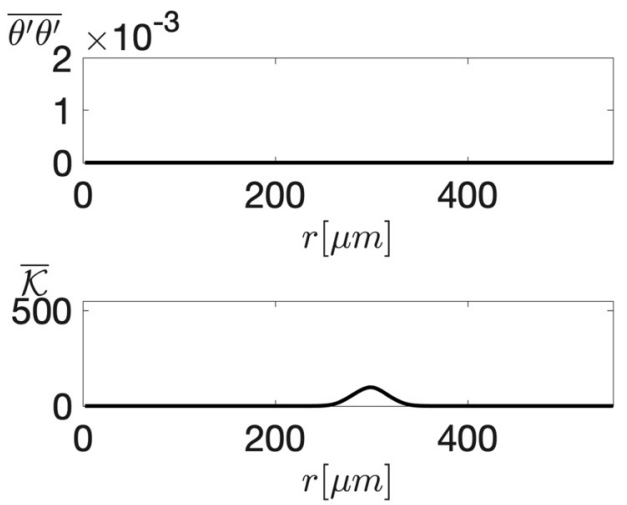

(a)
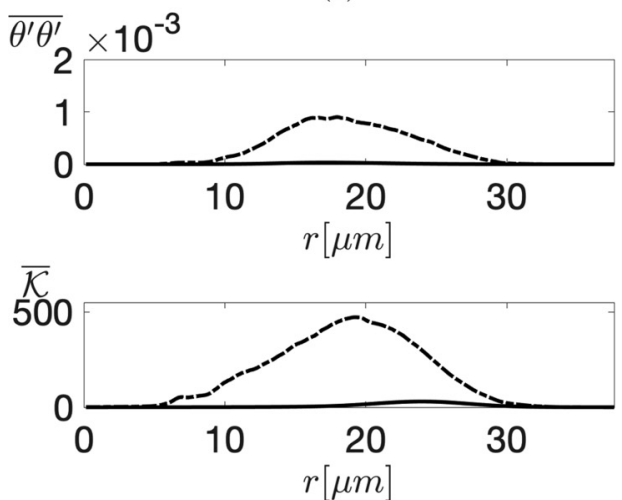

(c)
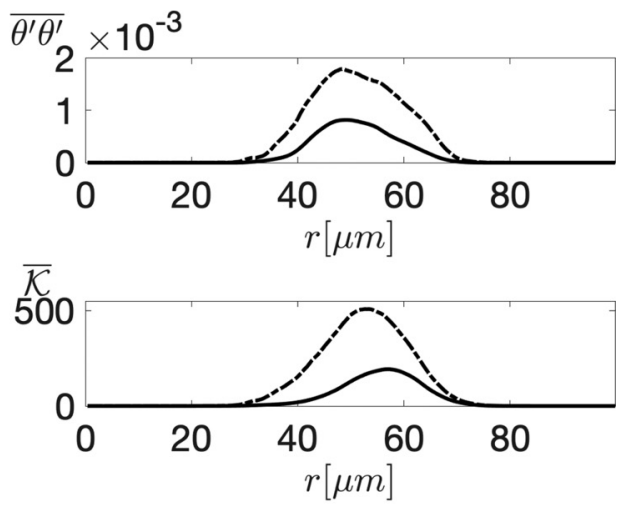

(b)
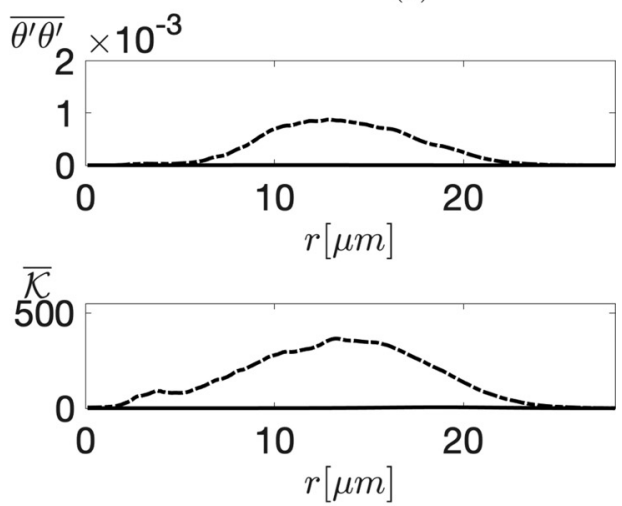

(d)

FIG. 7. Radial profiles of the variance of $\theta$ (top) and kinetic energy $\overline{\mathcal{K}}$ (bottom) at four times during compression. (a) Initial condition at $t=0 \mathrm{~ns}$ and $\Lambda=1$, (b) (II) $t=0.5 \mathrm{~ns}$ and $\Lambda=0.5$, (c) (III) $t=0.93 \mathrm{~ns}$ and $\Lambda=0.08$, and (d) (IV) $t=1 \mathrm{~ns}$ and $\Lambda=0.05$. Solid line for the $S 1_{a}$ simulation and dash-dotted line for the $S 1_{b}$ simulation.

phases identified in Fig. 4 in Sec. IV B. Whereas at early times there are small differences between simulations accounting or not for varying transport coefficients, at late times the sudden diffusion phase in $S 1_{a}$ marks a strongly different behavior. This phenomenon is very effective at contaminating the capsule center with heavy ablator elements.

In parallel, we present the mean profiles of turbulent kinetic energy $\overline{u_{i}^{\prime} u_{i}^{\prime}} / 2$ and variance $\overline{\theta^{\prime} \theta^{\prime}}$ in Fig. 7 also for $S 1_{a, b}$ simulations. These profiles are classically maximum at the center of the mixing layers. The relaminarization process due to viscous effects is marked by the dissipation of turbulent variances and occurs very soon, as shown by the differences between the simulations. Interestingly, relaminarization is close to symmetric between the fuel and ablator sides on $\overline{\theta^{\prime} \theta^{\prime}}$ radial profile, but this is not the case on kinetic energy profiles where dissipation seems to occur first on the DT side. Finally, only molecular diffusion is responsible for the sudden diffusion of the mixing layer as turbulent quantities are completely quenched at late times in $S 1_{a}$ simulation.

\section{Mixing parameter}

We further shed light on mixing in $S 1_{a, b}$ simulations using the molecular mixing parameter $\Xi(r, t)$ defined from the mass fraction of the ablator as (see Ref. [37]):

$$
\Xi(r, t)=\frac{\overline{Y(1-Y)}}{\bar{Y}(1-\bar{Y})}=1-\frac{\overline{Y^{\prime} Y^{\prime}}}{\bar{Y}(1-\bar{Y})} .
$$

The mixing parameter value thus reaches 1 when mixing is completed. This quantity is important in ICF for expressing the ratio between the amount of fusion reactions in the mixing zone and the amount obtained without mass fraction fluctuations [37]. Therefore, molecular mixing reflects to what extent the local mass fraction departs from the mean but does not give the relative amount of the two species in the mix [38].

We compare the temporal evolution of $\Xi$ between $S 1_{a}$ and $S 1_{b}$ simulations in Fig. 8. Here the mixing layer width is shown by the specific radii $r_{01}, r_{99}$, where $\bar{Y}=0.01$ reaches 0.01 and 0.99 , respectively. From time (I) to time (II)

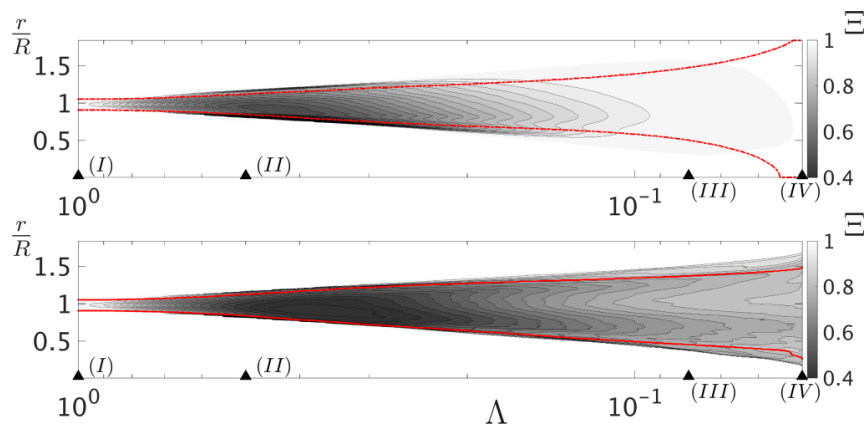

FIG. 8. Contour maps of the molecular mixing parameter $\Xi$ as a function of the compression parameter $\Lambda$ and normalized radial position $r / R$. Top: $S 1_{a}$ simulation; bottom: $S 1_{b}$ simulation. The red plain lines show the evolution of the mass fraction dependent radii $r_{01} / R$ and $r_{99} / R$, corresponding respectively to $\bar{Y}=0.01$ and $\bar{Y}=$ 0.99 . 


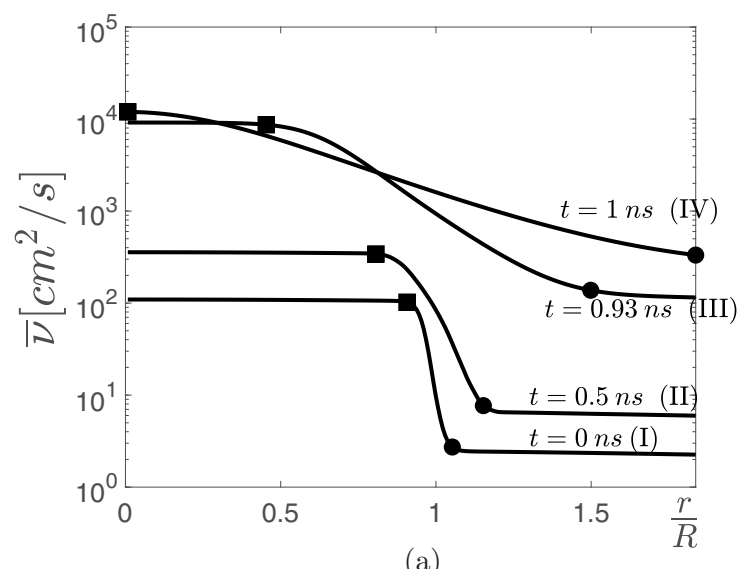

(a)

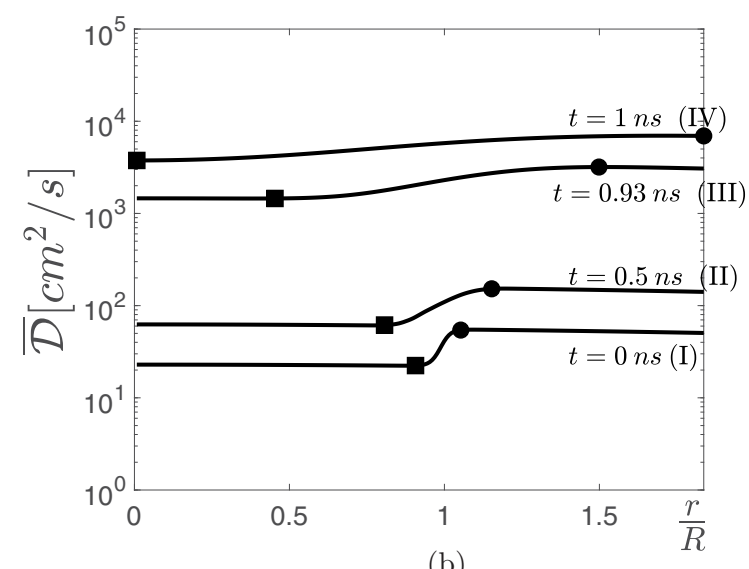

(b)

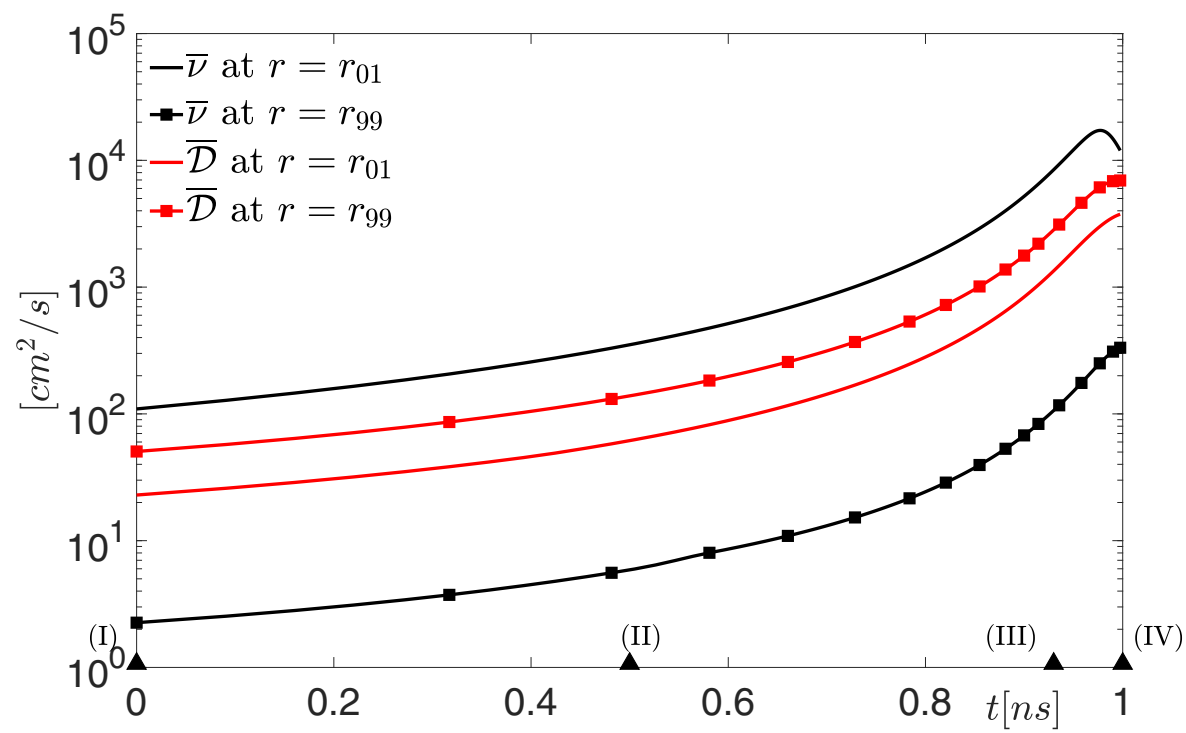

(c)

FIG. 9. Transport coefficient values extracted from $S 1_{a}$ simulation. [(a) and (b)] Mean radial profiles of viscosity and diffusion at four different instants. (c) Time evolution of mean viscosity and diffusion at the edges of the mixing layer at $r_{01}$ (solid line) and $r_{99}$ (dashed lines) corresponding to $Y=0.01$ and 0.99 , respectively. At the end of simulation ( $t \lesssim 1 \mathrm{~ns}$ ), $r_{01}$ reaches zero as mixing is entirely spread inside the capsule. The mean viscosity and diffusion are then plotted at the center of the capsule, $r=0$, and therefore decrease since $\bar{Y}$ becomes greater than 0.01 at $r=0$

corresponding to the rapid compression phase, the mixing parameter values are similar, since the process is mainly driven by turbulence.

From time (II) to time (III), although the sizes of the mixing layer are comparable between both simulations, the values of the molecular mixing parameter in $S 1_{a}$ become gradually larger than in $S 1_{b}$, due to the varying transport coefficients of the plasma. At time (III) and during the sudden diffusion phase, the values of $\Xi$ in $S 1_{a}$ become very close to 1 , indicating that the mixing is almost complete. By contrast in $S 1_{b}$ simulation, the mixing parameter also grows but at a smaller pace, as the mixing is driven by the turbulent cascade.

\section{E. Transport coefficient evolution}

In this section, we present the temporal and spatial evolutions of plasma transport coefficients used in the simulation and evaluated from the PIJ model [18-20].

We show in Fig. 9 the mean radial profiles of kinematic viscosity $\bar{v}(r, t)$ and diffusion $\overline{\mathcal{D}}(r, t)$ at different instants extracted from the $S 1_{a}$ simulation. The temporal evolutions of viscosity and diffusion are also shown for specific radii $r_{01}$ and $r_{99}$. Several classical features of transport coefficients in plasma are thus recovered. Viscosity and molecular diffusion follow the kinetic scaling laws [1] and thus experience a tremendous growth, up to two orders of magnitude, due to temperature increase. The radial mean profiles of $v$ exhibit a strong dependence on the mixture composition expressed by the mass fraction $Y$. The presence of heavy $\mathrm{CH}$ ions in the pure DT plasma indeed increases the effective plasma coupling parameter, leading to one- to two-orders-of-magnitude lower viscosity on the ablator side. Here the plasma coupling parameter indeed evolves from $3 \times 10^{-2}$ to $2 \times 10^{-3}$ in pure DT and from $3 \times 10^{-1}$ to $10^{-2}$ in pure $\mathrm{CH}$. Conversely, the molecular diffusion is 2-3 times higher on the ablator side. Indeed, the ion density number on the ablator side is lower than on the DT side, considering that the particle number distribution (ion and electron) $n$ is constant in the mixing layer. However, the spatial variations of $\mathcal{D}$ are clearly less 


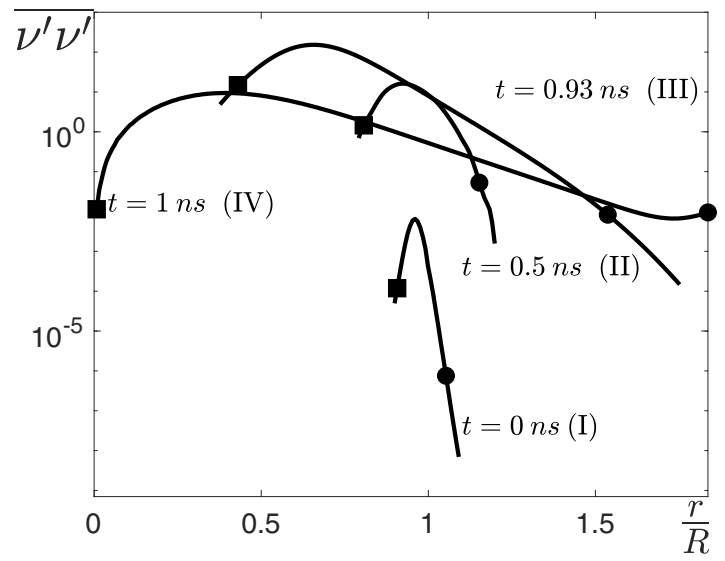

(a)

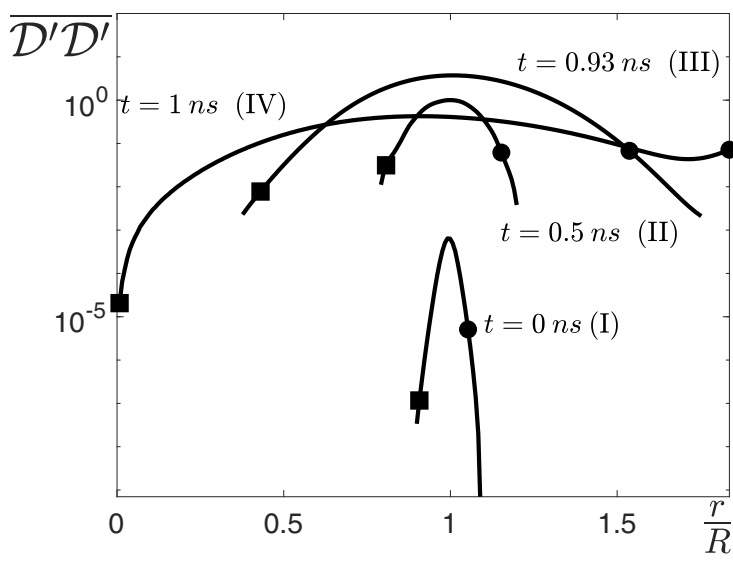

(b)

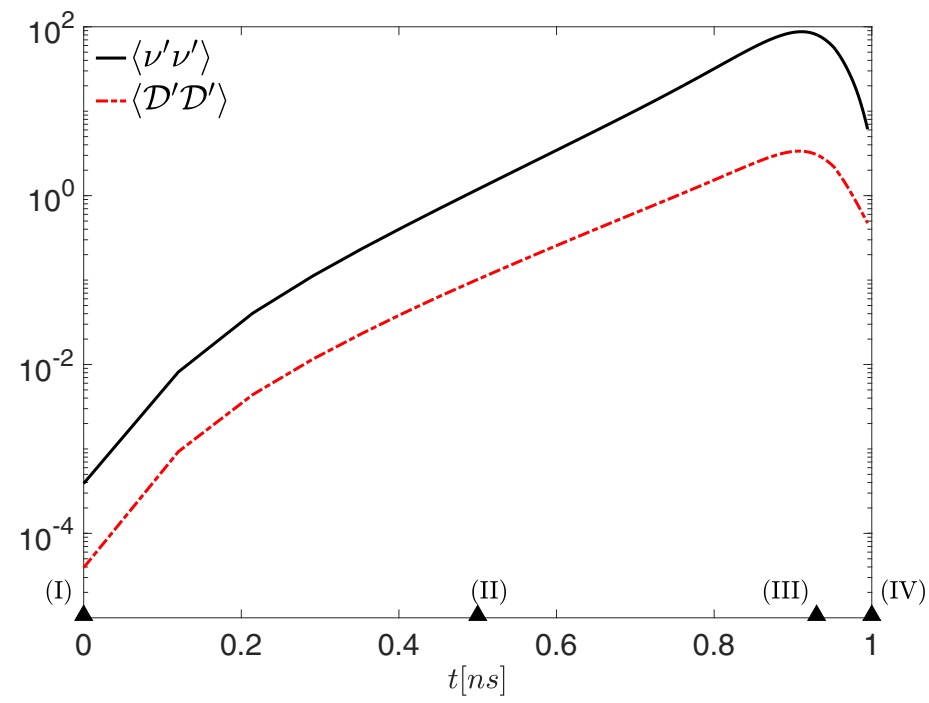

(c)

FIG. 10. Transport coefficient variances extracted from $S 1_{a}$ simulation. Top: Mean radial profiles of (a) viscosity, $\overline{v^{\prime} v^{\prime}}$, and (b) diffusion, $\overline{\mathcal{D}^{\prime} \mathcal{D}^{\prime}}$, at four different instants. (c) Time evolution of mean variance of viscosity $\left\langle v^{\prime} v^{\prime}\right\rangle$ and diffusion $\left\langle\mathcal{D}^{\prime} \mathcal{D}^{\prime}\right\rangle$.

spectacular than for viscosity. Consequently, the Schmidt number $v / \mathcal{D}$ is very high close to the DT side of the mixing layer, while of order unity on the $\mathrm{CH}$ side.

The time evolution of the mean variances of viscosity $\left\langle v^{\prime} v^{\prime}\right\rangle$ and diffusion, $\left\langle\mathcal{D}^{\prime} \mathcal{D}^{\prime}\right\rangle$, and their radial profiles, $\overline{v^{\prime} v^{\prime}}$ and $\overline{\mathcal{D}^{\prime} \mathcal{D}^{\prime}}$, are plotted at different instants in Fig. 10. These quantities reveal how fast viscosity and diffusion vary inside the mixing layer justifying the use of implicit iterative methods for numerical simulations. Transport coefficient variances increase during the compression until the decay of turbulence smooths the mass fraction fluctuations. Interestingly, while radial profiles of molecular diffusion variances remain maximum and well centered around the mixing layer center, $r / R=1$, viscosity variances shift toward the DT edge where even a small amount of $\mathrm{CH}$ drives huge variations of viscosity. This effect has also been observed on kinetic energy and $\theta^{\prime}$ variance profiles in Sec. IV C.

\section{F. Bidimensional maps}

In this section, we exhibit the instantaneous turbulent fields extracted from $S 1_{a, b}$ simulations to better understand how the relaminarization process and sudden diffusion effect induced by plasma transport coefficients operate.
We start by showing the two-dimensional contours of local kinetic energy $\mathcal{K}=u_{i} u_{i} / 2$ in $S 1_{a, b}$ simulations for the three radial positions, $r_{01}, r_{50}$, and $r_{99}$ (defined for $S 1_{b}$ ) and four times, (I), (II), (III), and (IV), in Fig. 11. The contour maps are obtained using the pseudocylindrical or Mollweide projection, as detailed in Appendix C.

Figure 11 clearly evidences the asymmetric relaminarization process due to the viscosity growth in $S 1_{a}$ simulation. The turbulent kinetic energy is first dissipated on the DT side of the mixing layer, following the spatial variations of viscosity as detailed in Sec. IV E. Also, the fact that turbulent structures are larger in $S 1_{a}$ simulation compared to $S 1_{b}$ simply shows that dissipation acts at small scales before reaching larger ones during the final phase of the compression. On the contrary, the constant viscosity of $S 1_{b}$ simulation allows the development of small structures by classical nonlinear energy cascade.

We now compare the structure of the $\theta^{\prime}$ variance in Fig. 12 at the center of the mixing zone, i.e., $r=r_{50}$, with kinetic energy contours at the same position. Interestingly, the characteristic sizes of the scalar field $\theta^{\prime} \theta^{\prime}$ are larger than for the kinetic energy contours, indicating that the dissipation is more effective for the variance of $\theta^{\prime}$ than for the turbulent kinetic energy, as already seen in Fig. 3. 


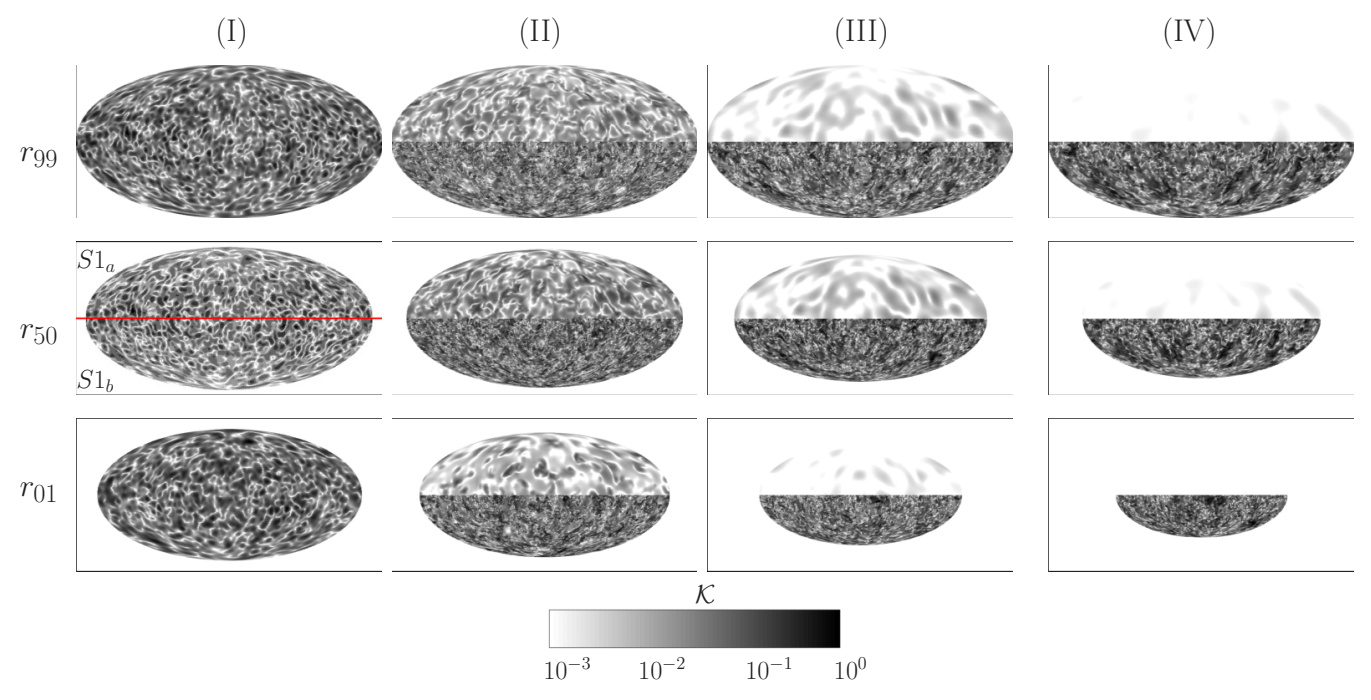

FIG. 11. Mollweide projection of the local turbulent kinetic energy in the mixing layer. The three rows correspond to the radial positions $r_{99}, r_{50}$, and $r_{01}$ defined in $S 1_{b}$ simulation, at times (I), (II), (III), and (IV). Top and bottom half contours corresponds respectively to $S 1_{a}$ and $S 1_{b}$ results.

\section{G. Spherical harmonics spectra}

The two-dimensional maps offer only a qualitative appreciation of the transport coefficient effects on the turbulence within the mixing layer. To quantify the information given by the maps of Sec. IV F, we use the spectral analysis of fluctuating fields on spherical surfaces proposed by Lombardini et al. [39]. The natural spectral basis to represent these fields are the spherical harmonics. For instance, a function $f\left(r_{i}, \psi, \phi\right)$ defined on a sphere of radius $r_{i}$ can be decomposed using the real spherical harmonic basis as

$$
f\left(r_{i}, \psi, \phi\right)=\sum_{l=0}^{\infty} \sum_{m=-l}^{l} f_{l m} Y_{l m}(\psi, \phi),
$$

where $Y_{l m}(\psi, \phi)$ are the real spherical harmonics [40,41] and $f_{l m}$ are the expansion coefficients (see also Appendix D).

Within this basis, it can be shown [39] that the angular power spectrum $C_{l}$ associated with the two-point correlation of the quantity $f$ taken on the sphere of radius $r_{i}$ (and assumed statistically homogeneous and isotropic on the same sphere) can be computed from the expansion coefficient as

$$
C_{l}=\frac{1}{2 l+1} \sum_{m=-l}^{l} f_{l m}^{2} .
$$

Considering the high $l$ spherical harmonics corresponds to very small characteristic dimensions with respect to sphere curvature, there is a direct relationship between the angular power spectra $C_{l}$ and the local one-dimensional planar spectrum $E(\kappa)$, where the "wave number" $\kappa$ is defined as $\kappa^{2}=l(l+1) / r_{i}^{2}$. In the limit of $l \gg 1, \kappa \simeq l / R$ and it can be shown that $l C_{l} \sim \kappa^{-\alpha}$ [39].

We thus compute the angular power spectra, using the field interpolated on spheres with radii $r_{01}, r_{50}$, and $r_{99}$. Because of the mixing, these radii vary during the compressions. Therefore, in order to compare the different spectra at different times and radii, we plot $l C_{l}$ as a function of the nondimensional wave number $q_{l}$, which is directly related to the spherical harmonics number $l$ by the relation $q_{l}=\frac{R}{r_{i}} \frac{l}{2 \pi}$.

(I)

(II)

(III)

(IV)

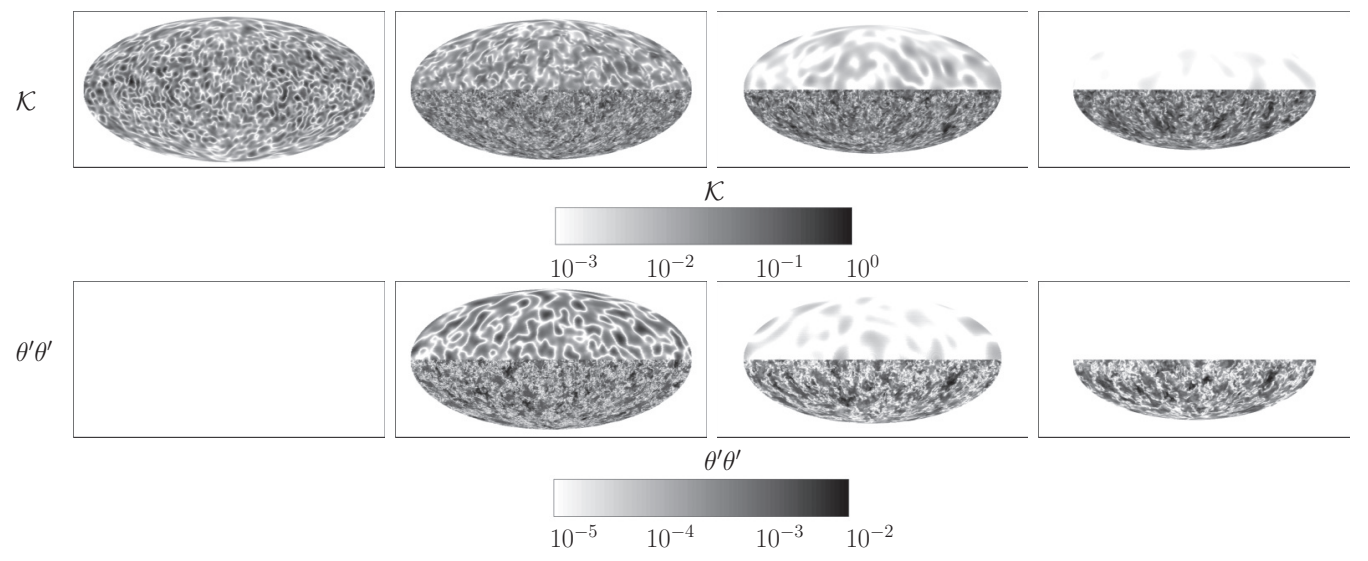

FIG. 12. Top: Mollweide projection of the kinetic energy. Bottom: Mollweide projection of the scalar variance. The radius of the spheres corresponds to the center of the mixing zone. Top and bottom half contours correspond respectively to $S 1_{a}$ and $S 1_{b}$ results. 

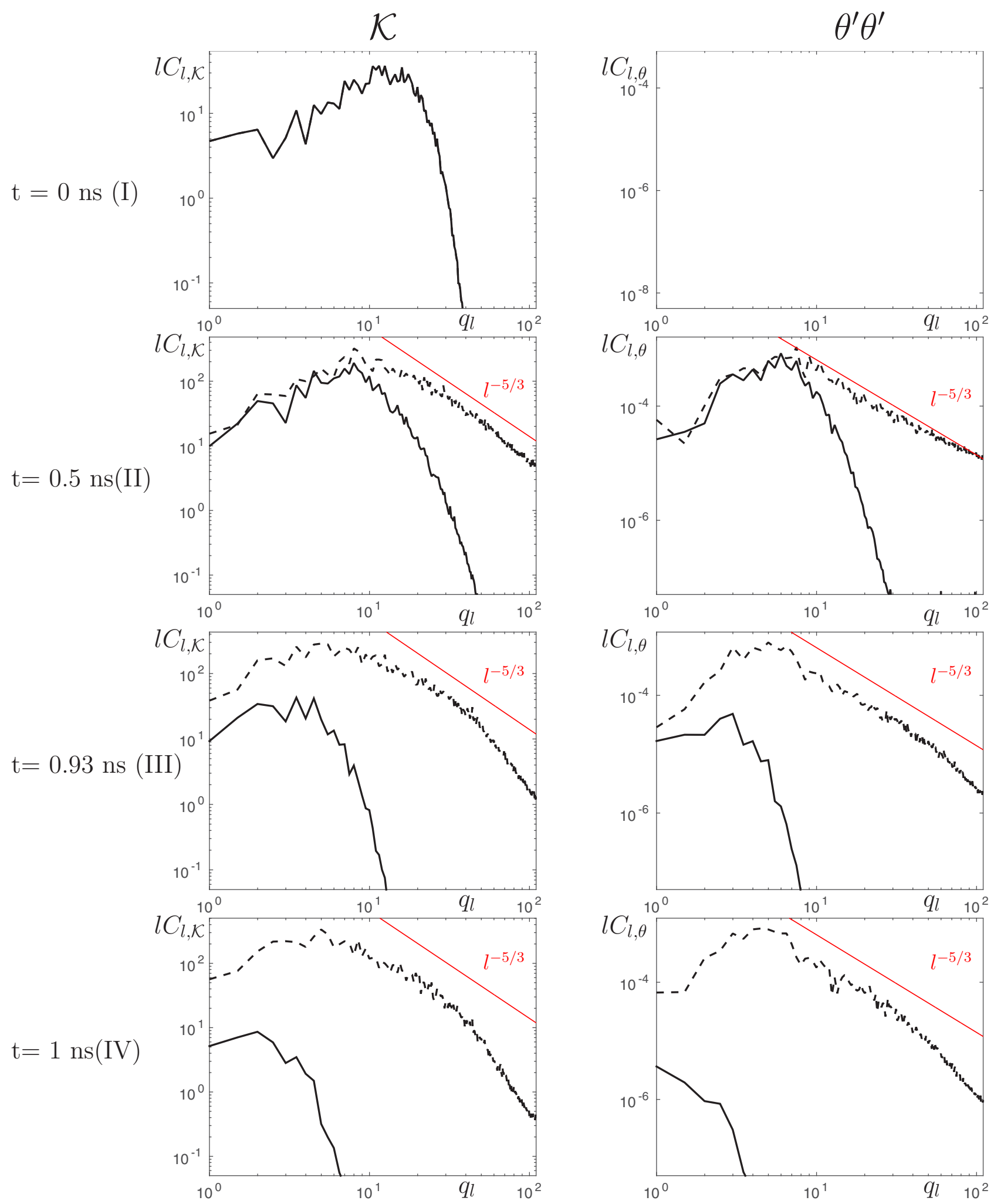

FIG. 13. Spherical harmonics spectra computed at the center of the mixing zone, $r_{50}$, at the instants I, II, III, and IV. Left: Kinetic energy spectra; right: Scalar spectra. Black solid line corresponds to the simulation $S 1_{a}$ and the dashed line to the simulation $S 1_{b}$. The red continuous line represents the $l^{-5 / 3}$ power law.

In Fig. 13, we confront the angular power spectra of kinetic energy $l C_{l, \mathcal{K}}$ and scalar field variance $l C_{l, \theta}$ between the simulations $S 1_{a}$ with varying plasma transport coefficients and $S 1_{b}$ with constant transport coefficients. At time (I), the kinetic energy spectra of the two initial conditions are superimposed, while, as we explained in Sec. III B, the initial conditions have no scalar fluctuations. At time (II), as observed on the bidimensional maps of Sec. IV F, in the constant viscosity 
simulation nonlinear phenomena produce a turbulent cascade. The inertial range, exhibiting a $l^{-5 / 3}$ slope of the kinetic energy spectrum, extends up to the maximum resolved $q_{l}$. On the contrary, the spectrum of the $S 1_{a}$ simulation shows less energetic scales, with no inertial range due to the increased value of viscosity. At small $q_{l}$, that coincides with the most energetic spherical harmonics, the two spectra have a similar energy distribution. This implies that the mixing zone evolution, in both simulations, is still driven by large-scale turbulent diffusion, confirming the results of Secs. IV B on the mixing zone width. The scalar spectra, $l C_{l, \theta}$, exhibit the same behavior, with the $l^{-5 / 3}$ scaling recovered for the constant transport coefficient simulations, and the relaminarization effects in the $S 1_{a}$ case. When the simulations reach time (III), $S 1_{b}$ kinetic energy spectrum maintains an inertial zone at intermediate wave number, but the $l^{-5 / 3}$ scaling does not extend to the $n_{l, \max }$, and we observe the beginning of a dissipative range. In contrast, for the $S 1_{a}$ case the energy-containing harmonics, for both kinetic energy and scalar, are reduced to the small $q_{l}$, suggesting that at this time the dynamics of the mixing zone is dominated entirely by viscous and diffusive effects. At time (IV) the $S 1_{a}$ spectra, for both quantities, show a very limited spherical harmonics range since the transport coefficients dissipate almost all the fluctuations. These results are consistent with the bidimensional maps of Sec. IV F where at time (IV) minimal fluctuations are visible. On the other hand, the constant coefficient spectra suggest that turbulence is still the primary driving phenomenon of the mixing zone evolution.

The temporal variation of the kinetic energy and scalar spectra of the $S 1_{a}$ simulation, in Fig. 14, is a further indication of the relaminarization caused by the transport coefficients.
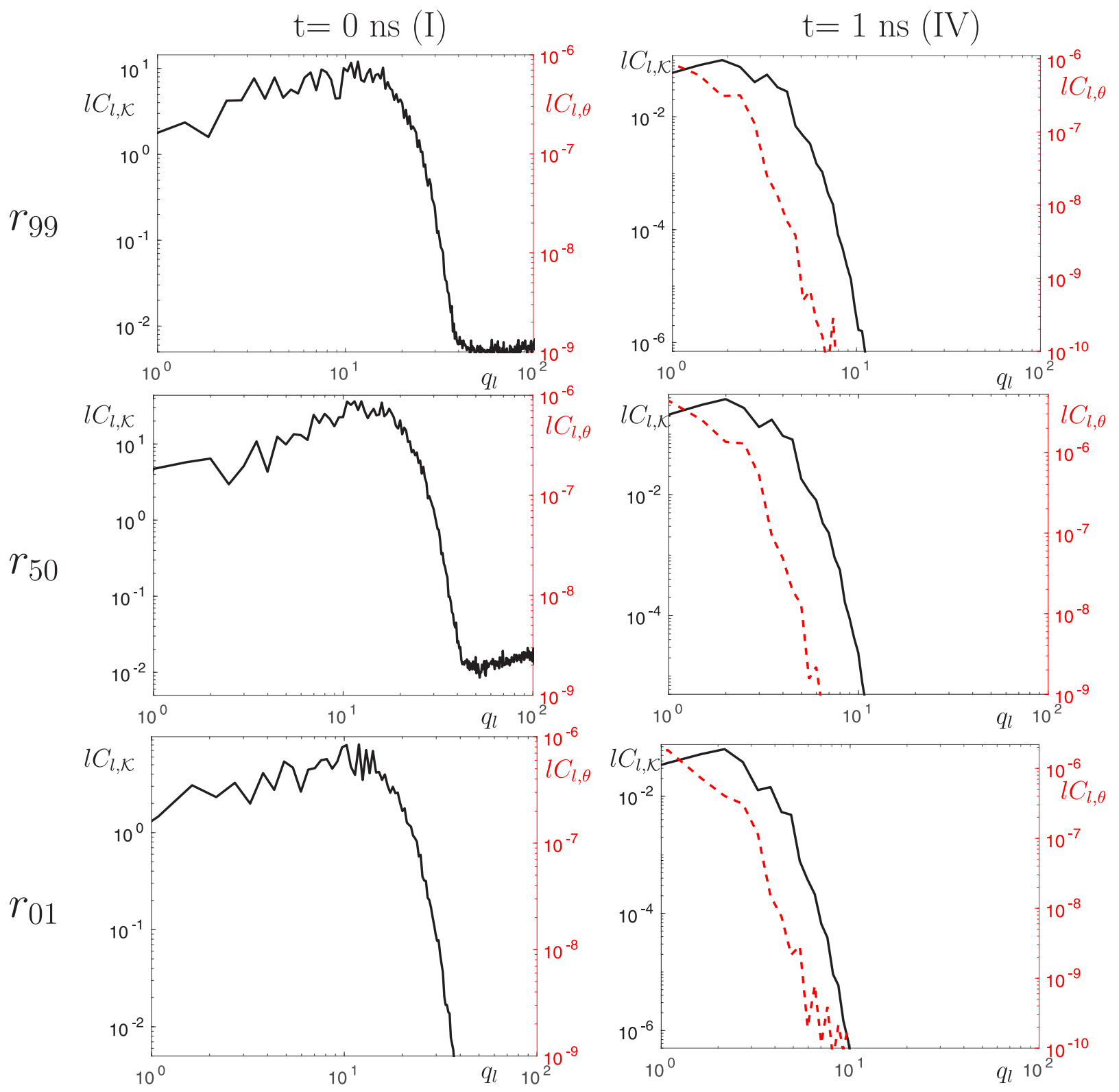

FIG. 14. Spherical harmonic spectra computed at the beginning (I) and at the end (IV) of the simulation $S 1_{a}$ at three radial positions $r_{01}$, $r_{50}, r_{99}$. Black solid lines correspond to kinetic energy spectra. Red dashed lines correspond to scalar spectra. 
This effect smooths equally velocity and density turbulent fluctuations, leaving only large-scale fluctuations at the end of the implosion.

\section{CONCLUSION}

This work presents numerical simulations of turbulent plasma mixtures under compression using a fluid approach and the PIJ [18-20] modeling of the transport coefficients, which vary with temperature, density and composition. The parameters are chosen to be representative of adiabatic implosions of DT-CH layers, mimicking the characteristic times and hot-spot thermodynamic conditions of an ICF capsule before the bang time. In order to achieve full resolution of low-Mach-number turbulent fluctuations, we use a Rogallo noninertial frame following the compression and the variable density approximation.

Simulations with or without varying plasma coefficients evidence the influence of plasma viscosity and molecular diffusion on the dynamics of mixing layers. A complex relaminarization process has been observed, occurring first on the DT side where viscosity is higher as shown by radial profiles and angular spectra. Although this phenomenon dissipates the small scales of turbulence and leads to a more homogeneous DT-CH mixing layer, it does not drastically reduce the dynamics of the mixing zone, mainly driven by larger scales. However, we show that during the late time evolution of the compression, the plasma molecular diffusion overcomes the turbulent one, leading to an enhanced diffusion of the DT-CH layer anticipated in Ref. [6]. This sudden diffusion effect comes along with the sudden viscous dissipation of turbulence already observed in Ref. [4].

The hot-spot contamination by heavy materials is crucial in the context of ICF as it leads to deleterious effects on the capsule yield. For configurations relevant to ignition, this study suggests that the DT layer placed at the ablator interface is important to prevent the diffusion of $\mathrm{CH}$ at the center of the capsule. Yet, for materials directly injected into the hot spot at early times, such as meteors from the filling tube [14], diffusion can occur very rapidly throughout the hot spot, depending on the temperature or density at the end of compression. This work also supports the hypothesis of mixing caused by physical diffusion in recent experiments, although not aimed at achieving ignition, performed at the Omega facility $[16,17]$. In addition, summing the plasma diffusion with the turbulent diffusion evaluated from turbulence models without low-Reynolds-number corrections appear an efficient strategy to quantify mixing at interfaces as the relaminarization process has a small impact on the layer dynamics.

\section{ACKNOWLEDGMENTS}

The authors are grateful to Dr. Marion Lafon for providing the thermodynamics conditions for Omega-type configurations. Simulations were performed at the computing center TGCC.

\section{APPENDIX A: ADDITIONAL CONFIGURATIONS RELEVANT TO ICF}

In this Appendix, we present additional results corresponding to six simulations with temperature, density, and final
TABLE III. Temperature $T$, pressure $P$, and density $\rho$ at the center of the capsule and at the beginning and end of simulation $N S$. The last column indicates the radius of the unperturbed fuel-ablator interface $R$.

\begin{tabular}{lccccc}
\hline \hline Name & $t(\mathrm{~ns})$ & $T(\mathrm{eV})$ & $P(\mathrm{Mbar})$ & $\rho\left(\mathrm{g} \mathrm{cm}^{-3}\right)$ & $R(\mu \mathrm{m})$ \\
\hline \multirow{2}{*}{$N S 1$} & 0 & 12.5 & $4.8 \times 10^{-2}$ & $5 \times 10^{-3}$ & 300 \\
& 1 & $5 \times 10^{3}$ & $1.52 \times 10^{5}$ & 40 & 15 \\
$N S 2$ & 0 & 12.5 & $4.8 \times 10^{-2}$ & $5 \times 10^{-3}$ & 300 \\
& 2 & $5 \times 10^{3}$ & $1.52 \times 10^{5}$ & 40 & 15 \\
\hline \hline
\end{tabular}

compression parameter close to the ones encountered in ICF experiments. In Appendix A 1 we present four compressions with the thermodynamic characteristics chosen to mimic an NIF implosion while in Appendix A 2 the two compressions studied have temperature and density similar to those found in Omega implosions.

\section{NIF-like configurations}

a. Implosions characteristics

In this section, we detail the results from two adiabatic compressions, having the same initial and final thermodynamic characteristics but which differ in the initial compression time rate $\mathcal{S}_{0}$. These conditions are gathered in Table III and in Fig. 15.

The initial conditions at $t=0$ correspond to a compressed and heated plasma state where DT and $\mathrm{CH}$ are already almost fully ionized with $Z_{\mathrm{DT}}=1$ and $Z_{\mathrm{CH}}=3.5$. The inner radius diameter is $R_{0}=300 \mu \mathrm{m}$, the duration of the compression is 1 or $2 \mathrm{~ns}$, and a convergence ratio of 20 is achieved. The base temperature at the center of the capsule varies from $12.5 \mathrm{eV}$ to $5 \mathrm{keV}$, with the base pressure reaching $150 \mathrm{Gbar}$ and fuel density $40 \mathrm{~g} \mathrm{~cm}^{-3}$.

\section{b. Initial conditions and simulations set-up}

We perform direct numerical simulations using the pseudospectral code described in Sec. III A. The initial conditions are generated with the method described in Sec. III B. With respect to a real NIF targets, in this case we do not consider the presence of the cryogenic DT layer between the $\mathrm{CH}$ and the gaseous DT. The simulations are characterized by an initial Reynolds number, $\operatorname{Re}_{0}=\frac{u_{0} \ell_{0}}{v}$, and the compression number $\mathrm{Cp}_{0}=\frac{u_{0}}{\ell_{0} \mathcal{S}_{0}}$. We chose for these simulations a Reynolds number of 16 , with a relatively lower level of turbulence than in the larger computations presented in the paper. The compression number are of the order of $0.1-0.05$, showing that the compression is relatively rapid compared to turbulence in the simulations. All the characteristics relative to the simulations are reported in Table IV.

\section{c. Mixing layer width}

We compare the time evolutions of the mixing layers sizes in Fig. 16 for simulations $N S 1_{a, b}$ (in black) and $N S 2_{a, b}$ (in red), with or without plasma effects. For both simulations $N S 1$ and $N S 2$, with low initial Reynolds number, we do not observe differences until the sudden diffusion phase at the end of the implosion. 


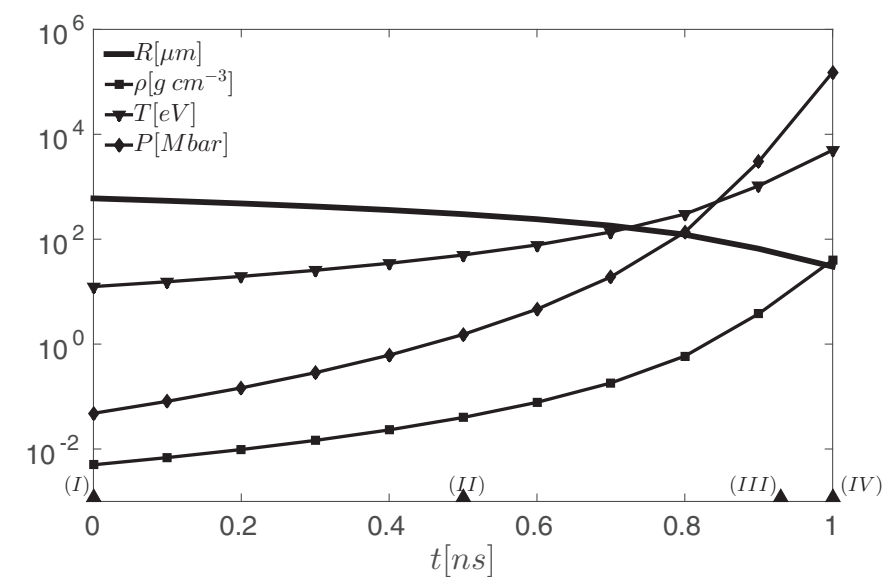

(a)

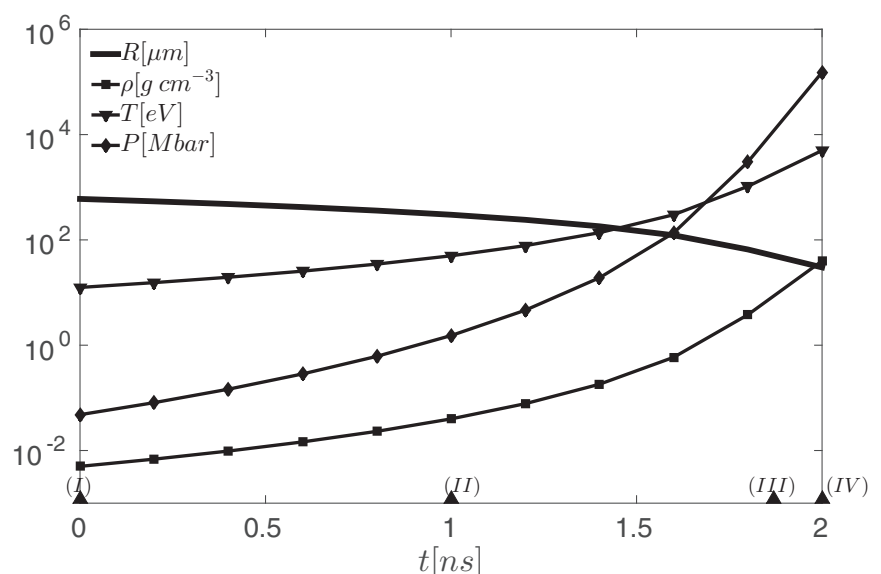

(b)

FIG. 15. Description of the implosion characteristics of the two NIF-like implosions. Time evolution of the temperature $T^{B}$, the pressure $P^{B}$, and density $\rho^{B}$ at the center of the domain. The thick solid line represents the radius of the capsule. (a) Simulations $N S 1$ and (b) simulations NS2.

\section{d. Two-dimensional contours}

With two-dimensional contours displayed in Figs. 17(a) and 17(b), we observe a lower diffusion than in the case presented in the paper. Nonetheless, a diffusion effect on the mixing zone is still present. We recall that in our simulation we do not consider the DT layer, which decrease the diffusion effects at the interface between DT and $\mathrm{CH}$.

\section{Omega-like configuration \\ a. Implosion characteristics}

In this section, we present the results from one compression relevant to Omega capsules. The thermodynamic conditions for these implosions are shown in Table $\mathrm{V}$ and in Fig. 18.

The initial inner radius diameter is $R_{0}=400 \mu \mathrm{m}$, the duration of the compression $0.42 \mathrm{~ns}$, and a convergence ratio of 10 is achieved. The base temperature at the center of the capsule varies from $110 \mathrm{eV}$ to $11 \mathrm{keV}$, with the base pressure reaching $0.8 \mathrm{Gbar}$ and fuel density $0.1 \mathrm{~g} \mathrm{~cm}^{-3}$.

TABLE IV. Simulation characteristics in terms of initial compression time rate $\mathcal{S}_{0}$, Reynolds number, compression number, integral length scale, and mixing layer size. As in the main paper, type $a$ corresponds to simulations with varying plasma transport coefficients while for type $b$ viscosity and molecular diffusion are kept constant during the computation.

\begin{tabular}{lcccccccc}
\hline \hline Name & & Type & Mesh size & $\mathcal{S}_{0}$ & $\mathrm{Re}_{0}$ & $\mathrm{Cp}_{0}$ & $\ell_{0} / R_{0}$ & $L_{0} / R_{0}$ \\
\hline \multirow{2}{*}{$N S 1$} & $a$ & DNS & $256^{3}$ & 1000 & 16 & 0.056 & 0.07 & 0.016 \\
& $b$ & DNS & $256^{3}$ & 1000 & 16 & 0.056 & 0.07 & 0.016 \\
\multirow{2}{*}{$N S 2$} & $a$ & DNS & $256^{3}$ & 500 & 16 & 0.1 & 0.07 & 0.016 \\
& $b$ & DNS & $256^{3}$ & 500 & 16 & 0.1 & 0.07 & 0.016 \\
\hline \hline
\end{tabular}

\section{b. Initial conditions and simulations set-up}

We perform direct numerical simulations using the pseudospectral code described in Sec. III A of the paper. The initial conditions are generated with the method described in Sec. III B. As in the previous section, the simulations are characterized by initial Reynolds and compression numbers. We use the same $\mathrm{Re}_{0}$ as in Appendix A 1 while the compression number $\mathrm{Cp}_{0}$ is lower due to the higher compression time rate $\mathcal{S}_{0}$. All the characteristics relative to the simulations are reported in Table VI. With respect to the case discussed by Zylstra et al. [17] in the simulations $\mathrm{ZS}_{a, b}$, the initial interface between DT and $\mathrm{CH}$ is more diffused, due to computational constraints.

\section{c. Mixing layer width}

We compare the time evolutions of the mixing layers sizes in Fig. 19, for simulations $Z_{a, b}$, with or without plasma effects. Unlike the NIF-like configuration of Appendix A 1,

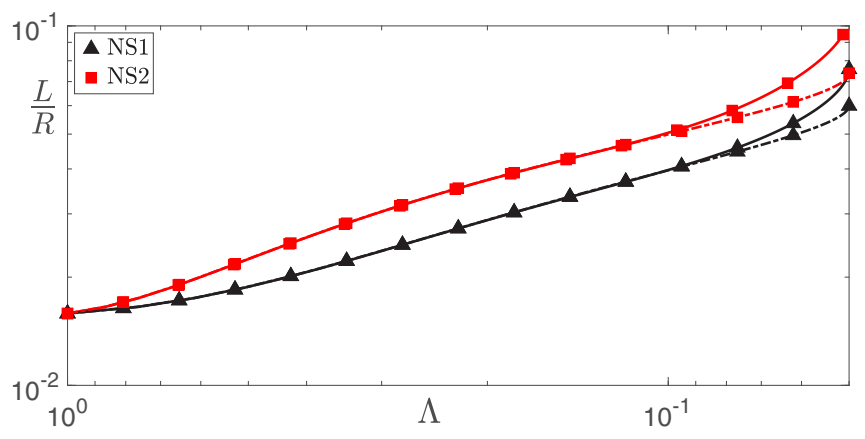

FIG. 16. Evolution of the renormalized mixing layer width as a function of the compression parameter $\Lambda$. The solid line represents the results of direct numerical simulations with plasma transport coefficients $a$, while the dashed line the case of constant transport coefficients $b$. The colors correspond to different simulations indicated in the legend. 


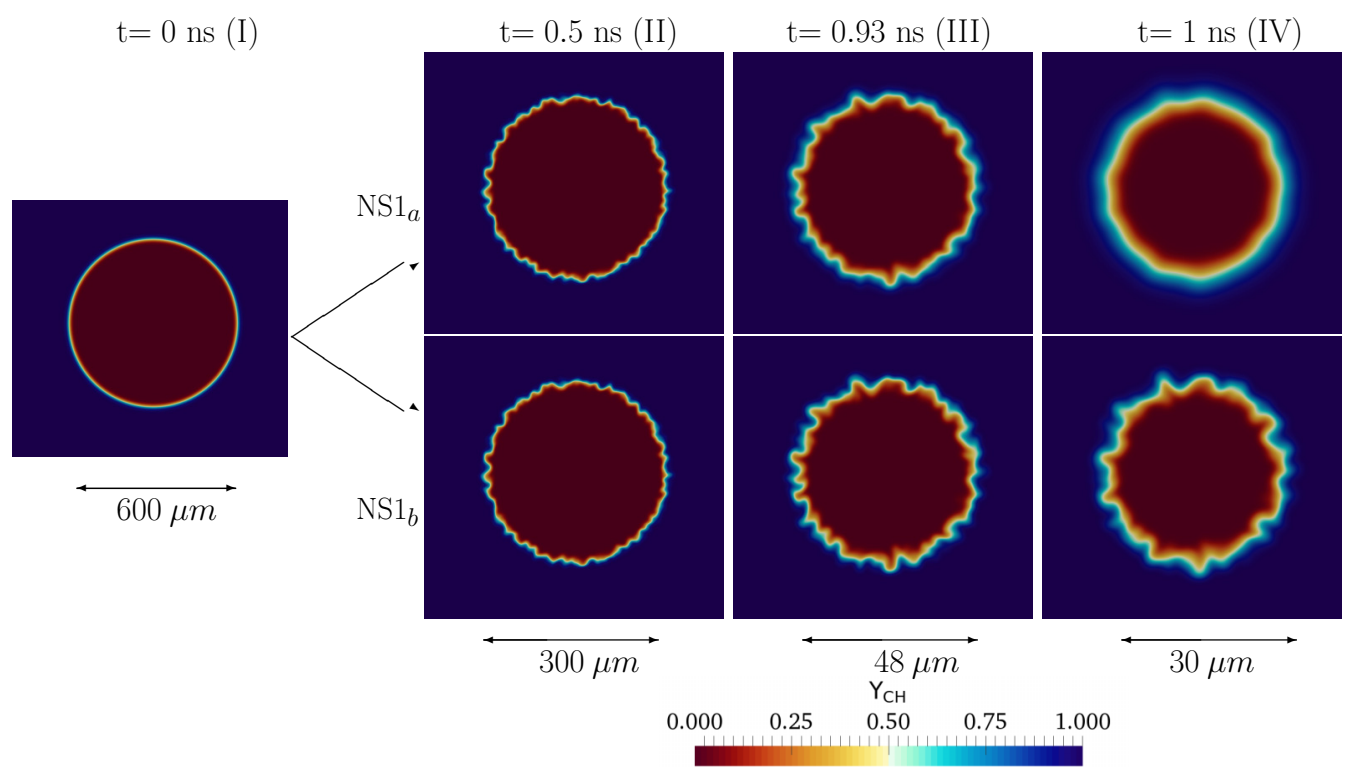

(a)

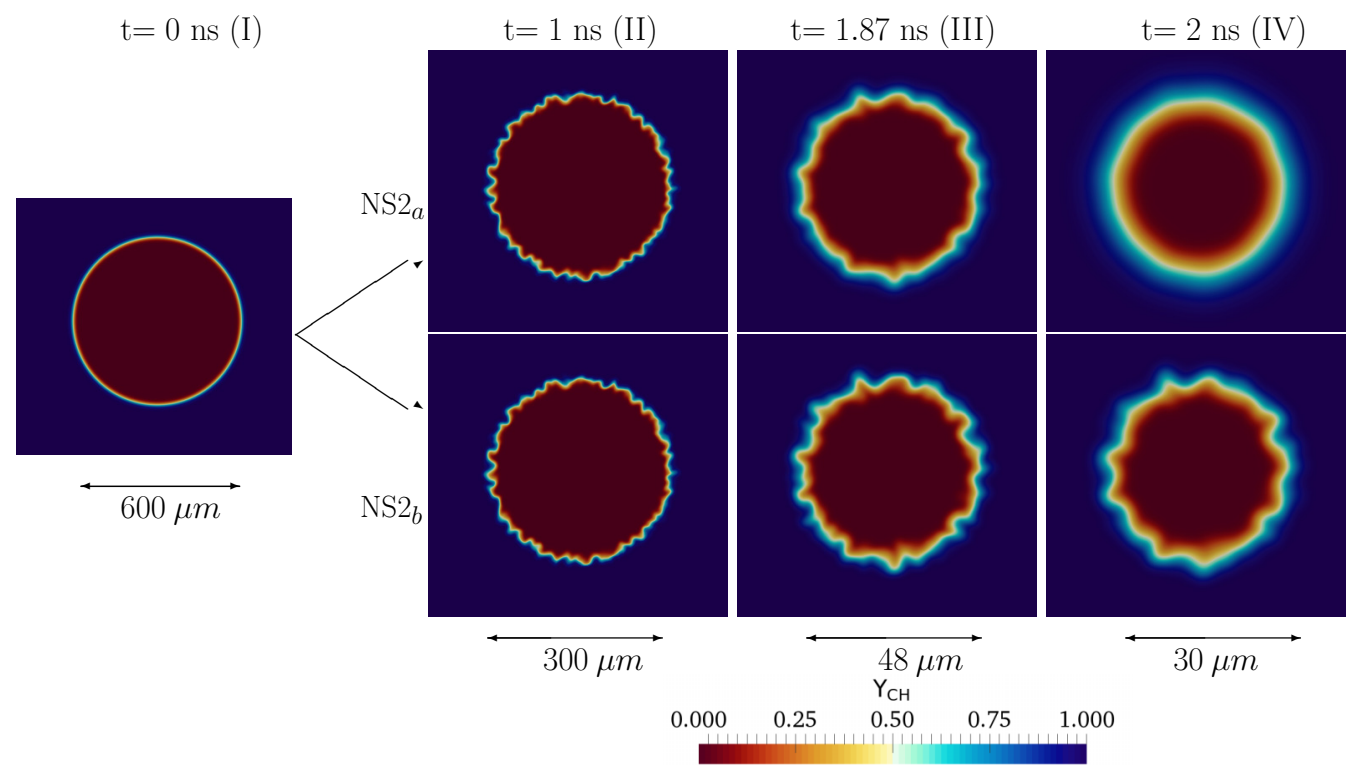

(b)

FIG. 17. Two-dimensional contours of $\mathrm{CH}$ mass fraction $Y$ at different instants and for simulations with varying $a$, and constant $b$, transport coefficients. (a) Results of NS1 simulations and (b) Results of NS2 simulations. The different times correspond to those of Fig. 15.

we observe a clear difference between simulations $\mathrm{ZS}_{a}$ and $\mathrm{ZS}_{b}$ starting with the first phase of the compression. In this case, the variation of transport coefficients becomes crucial

TABLE V. Temperature $T$, pressure $P$, and density $\rho$ at the center of the capsule and at the beginning and end of simulations. The last column indicates the radius of the unperturbed fuel-ablator interface $R$.

\begin{tabular}{lcccr}
\hline \hline$t(\mathrm{~ns})$ & $T(\mathrm{eV})$ & $P($ Mbar $)$ & $\rho\left(\mathrm{g} \mathrm{cm}^{-3}\right)$ & $R(\mu \mathrm{m})$ \\
\hline 0 & 110 & $8.4 \times 10^{-3}$ & $1 \times 10^{-4}$ & 400 \\
0.42 & $11 \times 10^{3}$ & $8.42 \times 10^{2}$ & $1 \times 10^{-1}$ & 40 \\
\hline \hline
\end{tabular}

early in the implosion, and their growth is an essential factor for fuel pollution.

TABLE VI. Simulation characteristics in terms of initial compression time rate $\mathcal{S}_{0}$, Reynolds number, compression number, integral length scale, and mixing layer size. Type $a$ corresponds to simulations with varying plasma transport coefficients while for type $b$ viscosity and molecular diffusion are kept constant during the computation.

\begin{tabular}{lcccccccc}
\hline \hline Name & & Type & Mesh size & $\mathcal{S}_{0}$ & $\mathrm{Re}_{0}$ & $\mathrm{Cp}_{0}$ & $\ell_{0} / R_{0}$ & $L_{0} / R_{0}$ \\
\hline \multirow{2}{*}{ ZS } & $a$ & DNS & $256^{3}$ & 2350 & 16 & 0.021 & 0.07 & 0.016 \\
& $b$ & DNS & $256^{3}$ & 2350 & 16 & 0.021 & 0.07 & 0.016 \\
\hline \hline
\end{tabular}




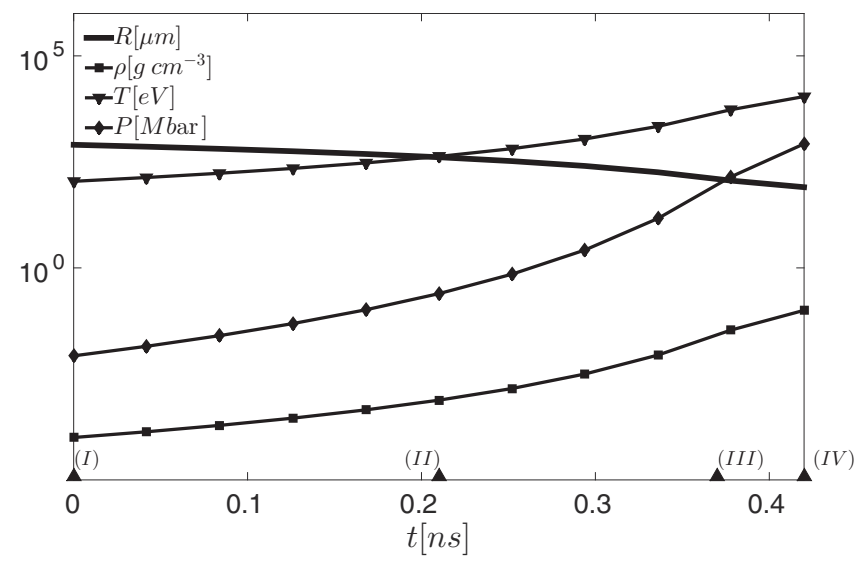

FIG. 18. Description of the implosion characteristics of the two Omega-like implosions. Time evolution of the temperature $T^{B}$, the pressure $P^{B}$, and density $\rho^{B}$ at the center of the domain. The thick solid line represents the radius of the capsule

\section{d. Two-dimensional contours}

The properties of the implosion evidenced by the mixing layer width evolution are confirmed by the two-dimensional contours of the $\mathrm{CH}$ mass fraction in Fig. 20.

\section{APPENDIX B: DESCRIPTION OF THE PSEUDO-ION IN JELLIUM MODEL}

Consider a plasma mixture made of $M$ components. Each component is made of $N_{i}$ ions of charge $Q_{i}$ and atomic mass $M_{i}$. Its number concentration is $X_{i}=N_{i} / N$, where the total number $N$ of atoms is $N=\sum_{i=1}^{M} N_{i}$. Noting $V$ as the total volume, the density of species $i$ is $n_{i}=N_{i} / V$, and the total density $n=N / V$.

\section{Interdiffusion}

We compute the interdiffusion coefficients using kinetic theory with an "excess" correction in the strongly coupled regime

$$
\mathcal{D}_{i j}=\mathcal{D}_{i j}^{\mathrm{kin}}+\Delta \mathcal{D}_{i j}^{\mathrm{ex}}
$$

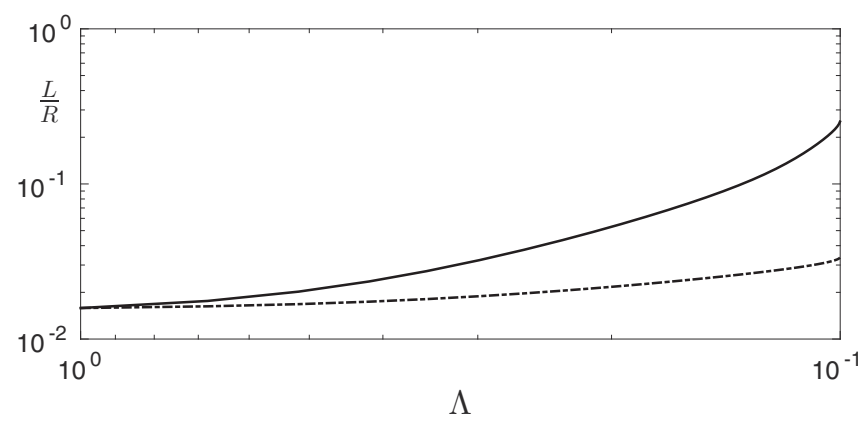

FIG. 19. Evolution of the renormalized mixing layer width as a function of the compression parameter $\Lambda$ for simulations ZS. The solid line represents the results of direct numerical simulations with varying plasma transport coefficients $Z_{a}$, while the dashed line the case of constant transport coefficients $\mathrm{ZS}_{b}$. with $[1,42]$

$$
\mathcal{D}_{i j}^{\mathrm{kin}}=R_{i j} \frac{X_{j}}{M_{i}} \frac{k_{b} T}{\omega_{i j}},
$$

where $\omega_{i j}$ is the collision frequency for species $i$, considering its collisions with species $j$, defined below. The factor $R_{i j}$, named "relaxation correction," may be used to improve the approximation. It represents the effect of the distortion of the Maxwellian distribution in the presence of concentration gradients and may vary from 1 to around 4 according to the mass ratios $M_{i} / M_{j}$ and the Coulomb coupling. In this version of PIJ, all the factors $R_{i j}$ equal 2 .

The excess correction is computed from the Darken relation,

$$
\Delta \mathcal{D}_{i j}^{\mathrm{ex}}=X_{i} \Delta \mathcal{D}_{j}^{\mathrm{ex}}+X_{j} \Delta \mathcal{D}_{i}^{\mathrm{ex}} .
$$

Indeed, molecular-dynamics simulations of binary ionic mixtures, in moderate to strong coupling, evidenced the accuracy of this relation, which reproduces the limit of extreme dilution of a component. In this limit, the self-diffusion of the impurity in the mixture represents the interdiffusion between this diluted species and the other components.

For each self-diffusion $\mathcal{D}_{i}$, equivalent OCPs are defined with coupling parameters $\Gamma_{i}=Q_{i}^{2} e^{2} / k_{B} T a_{i}$, corresponding to ions of charge $Q_{i}$ and Wigner-Seitz radius $a_{i}$

$$
\Delta \mathcal{D}_{i}^{\mathrm{ex}}=\mathcal{D}_{\mathrm{OCP}}\left(\Gamma_{i}\right)-\mathcal{D}_{\mathrm{OCP}}^{\mathrm{kin}}\left(\Gamma_{i}\right)
$$

The radius $a_{i}$ is the one of a sphere of uniformly distributed electrons that neutralize the charge $Q_{i}$, i.e., $\frac{4}{3} \pi a_{i}^{3} n_{e}=Q_{i}$, where the density of electrons in the mixture equals $n_{e}=$ $\sum_{i=1}^{M} Q_{i} n_{i}$

Reference [19] contains more details on the parametrizations of the OCP properties that are used in the PIJ model.

\section{Viscosity}

The bulk viscosity $\zeta$ vanishes in the weakly coupled regime [43] and is three orders of magnitude lower than the shear viscosity $\eta$ in the strongly coupled regime [44]. We shall therefore only consider $\eta$. We compute it using kinetic theory with an "excess" correction in the strongly coupled regime,

$$
\eta=\eta_{\mathrm{kin}}+\Delta \eta_{\mathrm{ex}}
$$

with $[1,42]$

$$
\eta_{\text {kin }}=0.965 \sum_{i=1}^{M} \frac{n_{i} k_{B} T}{\omega_{i}},
$$

where $\omega_{i}=\sum_{j=1}^{M} \omega_{i j}$ is a collision frequency for species $i$, considering its collisions with all the species present in the plasma. Viscosity is less sensitive to relaxation corrections and the coefficient 0.965 is the one of the OCP.

The excess correction uses an equivalent OCP with an effective coupling parameter for the whole mixture $\Gamma_{\text {eff }}=$ $\sum_{i=1}^{M} X_{i} \Gamma_{i}$,

$$
\Delta \eta_{\mathrm{ex}}=\eta_{\mathrm{OCP}}\left(\Gamma_{\mathrm{eff}}\right)-\eta_{\mathrm{OCP}}^{\mathrm{kin}}\left(\Gamma_{\mathrm{eff}}\right) .
$$




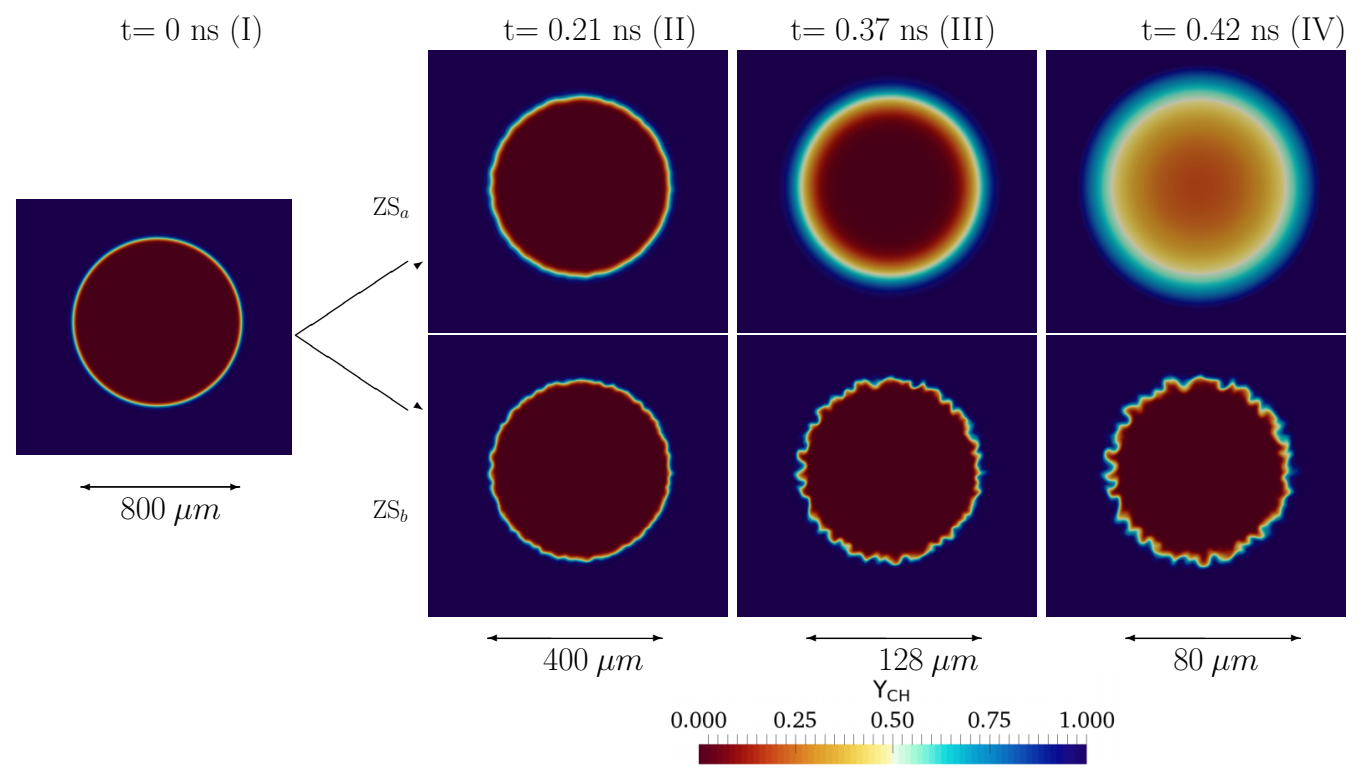

FIG. 20. Two-dimensional contours of mass fraction $Y$ of $\mathrm{CH}$ at different instants and for simulations with varying, $\mathrm{ZS}_{a}$, and constant, $\mathrm{ZS}_{b}$, viscosity and diffusion coefficients. The different times correspond to those of Fig. 18.

\section{Collision frequencies}

At the lowest order of approximation, the collision frequencies are given by a Maxwellian estimate. This estimate is obtained from the multifluid hydrodynamic equations, which correspond to the first three velocity moments of the kinetic equations. In these equations, the only collisional terms, which do not vanish for Maxwellian distribution functions $f_{i}(\mathbf{v})$ of species with equal temperature but different mean velocities $\mathbf{u}_{i}$, are the friction terms

$$
\mathbf{R}_{i j}=M_{i} \int\left(\mathbf{v}_{i}-\mathbf{u}_{i}\right) C_{i j}\left(f_{i}, f_{j}\right) d^{3} \mathbf{v}_{i},
$$

where $C_{i j}\left(f_{i}, f_{j}\right)$ is the collision operator of the kinetic equation. The Maxwellian estimate of $\omega_{i j}$ is defined by

$$
\mathbf{R}_{i j}=M_{i} n_{i} \omega_{i j}\left(\mathbf{u}_{j}-\mathbf{u}_{i}\right) .
$$

Using the Fokker-Planck-Landau collision operator in the limit of small relative velocity of the mixture components, $\left(\mathbf{u}_{j}-\mathbf{u}_{i}\right)^{2} \ll k_{b} T / M_{i j}$, where $M_{i j}=M_{i} M_{j} /\left(M_{i}+M_{j}\right)$ is the reduced mass, one gets [42]

$$
\omega_{i j}=\frac{n_{j}}{M_{i}} \frac{4 \sqrt{2 \pi M_{i j}} Q_{i}^{2} Q_{j}^{2} e^{4} \ln \Upsilon_{i j}}{3\left(k_{b} T\right)^{3 / 2}},
$$

where $\ln \Upsilon_{i j}$ is the Coulomb logarithm for binary collisions between species $i$ and $j$

$$
\ln \Upsilon_{i j}=\max \left[T_{i j}, \ln \left(\frac{b_{i j}^{\max }}{b_{i j}^{\min }}\right)\right]
$$

To avoid the pathological behavior when the argument of the logarithm becomes less than 1, we introduced the thresholds $T_{i j}$, taken equal to 3 in the current version. The minimum impact parameter, $b_{i j}^{\min }=Q_{i} Q_{j} e^{2} /\left(2 k_{b} T\right)$ and the maximum impact parameter, $b_{i j}^{\max }$, is given by the Debye screening length $\lambda_{D}$ for ions $\left(b_{i j}^{\max }\right)^{2}=k_{b} T /\left(4 \pi n\left\langle Q^{2}\right\rangle e^{2}\right)$, where $\left\langle Q^{2}\right\rangle=\sum_{i=1}^{M} X_{i} Q_{i}^{2}$

\section{APPENDIX C: MOLLWEIDE PROJECTION}

The Mollweide projection is an equal-area, pseudocylindrical map projection [45]. It has been previously used in other application for the projection of spherical results onto a plane such as the cosmic microwave background radiation [46].

The first step is to interpolate the field of interest on a sphere of given radius $R$ to get data as function of the polar angular coordinates $(\psi, \phi)$. The Mollweide projection establishes a relation between these variables and the map coordinates $X$ and $Y$. They represent respectively the equator and the central meridian,

$$
\begin{gathered}
X=R \frac{2 \sqrt{2}}{\pi} \psi \cos \lambda, \\
Y=R \sqrt{2} \sin \lambda,
\end{gathered}
$$

where $\lambda$ is a parametric angle defined by

$$
2 \lambda+2 \sin \lambda=\pi \sin \phi .
$$

Equations (C2) and (C3) have to be solved via an iterative Newton-Rhapson method.

\section{APPENDIX D: SPHERICAL HARMONICS}

We follow Lombardini et al. [39]. Here we sum up the principal steps:

(1) At time $t$ we have a field in the Cartesian reference frame $f(x, y, z)$. We interpolate it on a sphere of radius $R$ obtaining a $f(R, \theta, \phi)$.

(2) Using a discrete spherical harmonics transform we obtain the values $f_{l m}$. The spherical harmonics are defined by

$$
Y_{l m}(\theta, \phi)= \begin{cases}N_{(l, m)} P_{l}^{m}(\cos \theta) \cos (m \theta), & \text { if } m \geqslant 0 \\ N_{(l,|m|)} P_{l}^{|m|}(\cos \theta) \sin (|m| \theta), & \text { if } m<0\end{cases}
$$


where $P_{l}^{m}$ are the associated Legendre polynomials. The continuous spherical harmonics transform is defined as

$$
f_{l m}=\frac{1}{4 \pi} \iint_{\Omega} f(R, \theta, \phi) Y_{l m}(\theta, \phi) d \Omega
$$

The coefficient of the angular power spectrum can then be now computed as

$$
C_{l}=\frac{1}{2 l+1} \sum_{m=-l}^{m=+l}\left|f_{l m}\right|^{2} .
$$

[1] S. I. Braginskii, Rev. Plasma Phys. 1, 205 (1995).

[2] B. M. Haines, E. L. Vold, K. Molvig, C. Aldrich, and R. Rauenzahn, Phys. Plasmas 21, 092306 (2014).

[3] C. R. Weber, D. S. Clark, A. W. Cook, L. E. Busby, and H. F. Robey, Phys. Rev. E 89, 053106 (2014).

[4] S. Davidovits and N. J. Fisch, Phys. Rev. Lett. 116, 105004 (2016).

[5] A. Campos and B. E. Morgan, Phys. Rev. E 99, 013107 (2019).

[6] G. Viciconte, B.-J. Gréa, and F. S. Godeferd, Phys. Rev. E 97, 023201 (2018).

[7] S. Davidovits and N. J. Fisch, Phys. Rev. E 94, 053206 (2016).

[8] A. Campos and B. E. Morgan, Phys. Rev. E 99, 063103 (2019).

[9] R. Betti and O. Hurricane, Nat. Phys. 12, 435 (2016).

[10] T. Ma, P. Patel, N. Izumi, P. Springer, M. Key, L. Atherton, M. Barrios, L. Benedetti, R. Bionta, E. Bond et al., Phys. Plasmas 24, 056311 (2017).

[11] B. M. Haines, G. P. Grim, J. R. Fincke, R. C. Shah, C. J. Forrest, K. Silverstein, F. J. Marshall, M. Boswell, M. M. Fowler, R. A. Gore et al., Phys. Plasmas 23, 072709 (2016).

[12] B. Hammel, S. Haan, D. Clark, M. Edwards, S. Langer, M. Marinak, M. Patel, J. Salmonson, and H. Scott, High Energy Density Phys. 6, 171 (2010).

[13] A. G. MacPhee, D. T. Casey, D. S. Clark, S. Felker, J. E. Field, S. W. Haan, B. A. Hammel, J. Kroll, O. L. Landen, D. A. Martinez, P. Michel, J. Milovich, A. Moore, A. Nikroo, N. Rice, H. F. Robey, V. A. Smalyuk, M. Stadermann, and C. R. Weber, Phys. Rev. E 95, 031204(R) (2017).

[14] C. Weber, D. Casey, D. Clark, B. Hammel, A. MacPhee, J. Milovich, D. Martinez, H. Robey, V. Smalyuk, M. Stadermann et al., Phys. Plasmas 24, 056302 (2017).

[15] E. L. Vold, A. S. Joglekar, M. I. Ortega, R. Moll, D. Fenn, and K. Molvig, Phys. Plasmas 22, 112708 (2015).

[16] H. G. Rinderknecht, H. Sio, C. K. Li, A. B. Zylstra, M. J. Rosenberg, P. Amendt, J. Delettrez, C. Bellei, J. A. Frenje, M. Gatu Johnson et al., Phys. Rev. Lett. 112, 135001 (2014).

[17] A. B. Zylstra, N. M. Hoffman, H. W. Herrmann, M. J. Schmitt, Y. H. Kim, K. Meaney, A. Leatherland, S. Gales, C. Forrest, V. Y. Glebov, M. Schoff, M. Hoppe, and N. Ravelo, Phys. Rev. E 97, 061201(R) (2018).

[18] C. Ticknor, J. D. Kress, L. A. Collins, J. Clérouin, P. Arnault, and A. Decoster, Phys. Rev. E 93, 063208 (2016).

[19] P. Arnault, High Energy Density Phys. 9, 711 (2013).

[20] A. J. White, L. A. Collins, J. D. Kress, C. Ticknor, J. Clérouin, P. Arnault, and N. Desbiens, Phys. Rev. E 95, 063202 (2017).
[21] K. Molvig, A. N. Simakov, and E. L. Vold, Phys. Plasmas 21, 092709 (2014).

[22] G. Kagan and X.-Z. Tang, Phys. Lett. A 378, 1531 (2014).

[23] R. S. Rogallo, Tech. Rep. 81315, NASA, 1981.

[24] S. Atzeni and J. Meyer-ter Vehn, The Physics of Inertial Fusion (Oxford Science Publication, 2004).

[25] D. L. Sandoval, Ph.D. thesis, University of Washington (1995).

[26] D. Livescu and J. R. Ristorcelli, J. Fluid Mech. 591, 43 (2007).

[27] C. Cambon, Y. Mao, and D. Jeandel, Eur. J. Mech. B-Fluids. 11, 683 (1992).

[28] S. A. Orszag and G. S. Patterson, Phys. Rev. Lett. 28, 76 (1972).

[29] S. Gottlieb, C.-W. Shu, and E. Tadmor, SIAM Rev. 43, 89 (2001).

[30] D. Chung and D. I. Pullin, J. Fluid Mech. 643, 279 (2010).

[31] B. Dipierro and M. Abid, Eur. Phys. J. B 85, 69 (2012).

[32] J. Stoer and R. Bulirsch, Introduction to Numerical Analysis (Springer Science \& Business Media, New York, 2013), Vol. 12.

[33] B. J. R. Thornber and D. Drikakis, Int. J. Numer. Methods Fluids 56, 1535 (2008).

[34] F. F. Grinstein, L. G. Margolin, and W. J. Rider, Implicit Large Eddy Simulation: Computing Turbulent Fluid Dynamics (Cambridge University Press, Cambridge, 2007).

[35] M. J. Andrews and D. B. Spalding, Phys. Fluids A 2, 922 (1990).

[36] B.-J. Gréa, Phys. Fluids 25, 015118 (2013).

[37] D. L. Youngs, Phys. Fluids A 3, 1312 (1991).

[38] P. Danckwerts, Appl. Sci. Res., Sec. A 3, 279 (1952).

[39] M. Lombardini, D. Pullin, and D. Meiron, J. Fluid Mech. 748, 113 (2014).

[40] M. Abramowitz and I. Stegun, Handbook of Mathematical Functions (Dover, London, 1965).

[41] H. Groemer, Geometric Applications of Fourier Series and Spherical Harmonics (Cambridge University Press, Cambridge, 1996), Vol. 61.

[42] A. Decoster, P. A. Markowich, B. Perthame, and P.-A. Raviart, Modeling of Collisions (Elsevier, Amsterdam 1998), Vol. 2.

[43] L. Landau, E. Lifshitz, and L. Pitaevskij, Course of Theoretical Physics, Vol. 10: Physical Kinetics (Oxford University Press, Oxford, 1981).

[44] B. Bernu and P. Vieillefosse, Phys. Rev. A 18, 2345 (1978).

[45] J. P. Snyder, Map Projections-A Working Manual (US Government Printing Office, Washington DC, 1987), Vol. 1395.

[46] C. Bennett, D. Larson, J. Weiland, N. Jarosik, G. Hinshaw, N. Odegard, K. Smith, R. Hill, B. Gold, M. Halpern et al., Astrophys. J. Suppl. Ser. 208, 20 (2013). 